\title{
A guide through the computational analysis of isotope-labeled mass spectrometry-based quantitative proteomics data: an application study
}

Stefan P Albaum ${ }^{1,2^{*}}$, Hannes Hahne ${ }^{4,5,7}$, Andreas Otto ${ }^{5}$, Ute Haußmann ${ }^{6}$, Dörte Becher ${ }^{5}$, Ansgar Poetsch ${ }^{6}$, Alexander Goesmann ${ }^{1,3}$ and Tim W Nattkemper ${ }^{2}$

\begin{abstract}
Background: Mass spectrometry-based proteomics has reached a stage where it is possible to comprehensively analyze the whole proteome of a cell in one experiment. Here, the employment of stable isotopes has become a standard technique to yield relative abundance values of proteins. In recent times, more and more experiments are conducted that depict not only a static image of the up- or down-regulated proteins at a distinct time point but instead compare developmental stages of an organism or varying experimental conditions.

Results: Although the scientific questions behind these experiments are of course manifold, there are, nevertheless, two questions that commonly arise: 1) which proteins are differentially regulated regarding the selected experimental conditions, and 2) are there groups of proteins that show similar abundance ratios, indicating that they have a similar turnover? We give advice on how these two questions can be answered and comprehensively compare a variety of commonly applied computational methods and their outcomes.

Conclusions: This work provides guidance through the jungle of computational methods to analyze mass spectrometry-based isotope-labeled datasets and recommends an effective and easy-to-use evaluation strategy. We demonstrate our approach with three recently published datasets on Bacillus subtilis [1,2] and Corynebacterium glutamicum [3]. Special focus is placed on the application and validation of cluster analysis methods. All applied methods were implemented within the rich internet application QuPE [4]. Results can be found at http://qupe. cebitec.uni-bielefeld.de.
\end{abstract}

\section{Background}

Developments in the field of mass spectrometry over the last decade have brought the analysis of proteins to a new level, and allow today's scientists to comprehensively scrutinize these integral components of life that act as molecular machines, structural elements, transporters, or receptors [5]. In high-throughput experiments, liquid chromatography coupled to tandem mass spectrometry (LC-MS/MS) is utilized to characterize the complete set of proteins contained in a cell or organism. Recent methods, moreover, employ isotopic labels to

\footnotetext{
* Correspondence: alu@cebitec.uni-bielefeld.de

'Computational Genomics, Center for Biotechnology (CeBiTec), Bielefeld University, Germany

Full list of author information is available at the end of the article

enable the quantification of proteins [6-14]. Datasets resulting from such quantitative proteomics experiments are often very complex and consist of lists of measured abundance values for hundreds (or thousands) of proteins. As a manual exploration of such large datasets is practically impossible, there is a strong need for computational approaches concerning statistical data analysis and data mining in order to support experimenters. The scientific questions being addressed by these experiments are without any doubt very different. There are, however, two questions that commonly arise: 1 ) which proteins are differentially regulated regarding the selected experimental conditions, and 2) are there groups of proteins that are characterized by similar abundance ratios, indicating a common regulation? Aim 
of this work is to answer these two questions considering-as application example-three real-world datasets on Bacillus subtilis [1,2] and Corynebacterium glutamicum [3], thereby taking into account the particular challenges of mass spectrometry-based proteomics data.

\section{Question 1)}

Obviously, each time a measurement of a protein's abundance is performed, an-albeit small-variation in the recorded value may occur. This variation may have different origins, and that is what needs to be determined: are the changes governed by regulatory mechanisms in a cell, e. g. as a response to a stress stimulus an organism is exposed to, or do they originate from other sources such as a natural fluctuation or technical errors in the process of measurement itself. Given a number of measured abundance ratios for a protein, a small variation between these values could mean that the strict enforcement of the protein's quantity is of key importance, e. g. for the development of an organism. Contrary, a rather high variation could indicate a weak influence of regulatory elements and lead to the assumption that the exact dosage e. g. of an enzyme regarding a metabolic pathway may not be important. If, for a protein, repeated measurements are obtained under different conditions, $i$. e. can be separated into two or more groups, it can be questioned whether variations are larger between two groups than within the same group. In order to assess the significance of deviations a statistical test such as the analysis of variance (ANOVA) may be employed. A meaningful interpretation of the results, however, demands certain prerequisites: i) within a group, deviations from the group's mean value should follow a Gaussian-distribution, ii) the samples should be taken from equally distributed populations; therefore, variances within different samples are not allowed to differ significantly, and iii) the influence of confounding variables has to be independent for each measurement. Infringements of these premises, in particular of ii, might result in the false assessment of proteins as significantly differentially regulated. Although the ANOVA has more power in terms of discovering significant differences, in cases of violated assumptions a non-parametric method such as the Kruskal-Wallis one-way analysis of variance has to be applied $[15,16]$.

\section{Question 2)}

In the analysis of these complex datasets one is often interested in the determination of protein groups that show similar changes in abundance in relation to the experimental conditions. It seems reasonable to suppose that these proteins are commonly regulated or functionally related. A computational method to identify groups of proteins with similar abundance profiles is cluster analysis. Belonging to the group of unsupervised learning methods, cluster analysis is characterized in that no external information is needed. The operation is solely performed on inherent features of the data-clusters are not known a priori but discovered during the clustering process. The aim of clustering is to aggregate a number of measurements, i. e. proteins, in groups, so called clusters, such that all members of a group are as homogeneous as possible, while at the same time requiring that there is a considerable heterogeneity between all elements of two clusters $[17,18]$. Clustering techniques are traditionally divided into three distinct classes: a) hierarchical, b) partitioning or vector quantization, and c) probabilistic or density-based methods [19,20]. a) (Agglomerative) hierarchical approaches group objects into clusters, which in turn are iteratively grouped into clusters, thereby forming a hierarchical tree structure [21-23]. b) Following a given optimization strategy and a specified number of groups partitioning approaches assign each individual to one distinct group. One of the most prominent algorithms is K-means [24,25]. c) Density-based approaches differ from the other two strategies in the way that each object not necessarily belongs to a single cluster but instead is assigned a probability that specifies its membership to a group. An example is fuzzy C-means clustering [26].

Cluster analysis has the potential to reveal hidden structures in the data, which-in the context of quantitative proteomics-might be groups of proteins having a similar pattern of regulation. However, the validity of the outcome of an unsupervised learning method such as cluster analysis is difficult to assess (cf. i.a. [18]). In the run-up to the analysis, in general, no information regarding a true clustering is available. Moreover, the results produced by different algorithms are (very) often dissimilar: the hierarchical structures for example obtained by Single- and Complete-linkage are seldom characterized by a strong congruence. A fundamental part of the clustering process therefore is an evaluation of the algorithm's results $[27,28]$, which, to our knowledge, has been discussed for other "omics"-data but so far not for quantitative proteomics datasets.

\section{Results and Discussion}

Our study is based on three real-world datasets. Two experiments on Bacillus subtilis consist of each three biological replicate measurements, and describe a time series of five distinct time points. In experiment A, samples were taken directly after a salt stress was induced and after 10, 30, 60, and 120 minutes [1]. In experiment $B$, which unveils temporal changes in the proteome caused by glucose starvation, cells were harvested during exponential growth, and 0, 30, 60, and 120 minutes after transition from exponential to stationary growth phase 
[2]. A third experiment $C$ investigates the adaption of Corynebacterium glutamicum to alternative carbon sources [3]. In contrast to the aforementioned experiments, two different growth media-benzoate and glucose-were examined. It was, moreover, decided to include only one replicate in this analysis to demonstrate the applicability of the provided evaluation strategy on smaller datasets. Please note therefore that the following analysis results of this experiment are not comparable to the results presented in the original, very comprehensive proteomics study. The two questions to answer in all three experiments are: 1) which proteins are differentially regulated regarding the factor time $(\mathrm{A}, \mathrm{B})$ or, in case of experiment $\mathrm{C}$, regarding the factor carbon source, and 2) are there groups of proteins that show a similar pattern of regulation in terms of their relative abundance.

For experiment A and B, Mascot (TM) [29] was used for protein identification, for experiment $C$ existing identifications resulting from Sequest (TM) [30] were imported in QuPE [4]. After quantification using QuPE's built-in algorithm, in experiment $\mathrm{A}$ abundance ratios had been calculated for 58,895 peptides leading to 1,285 different quantified proteins with at least one measurement for at least one time point; in experiment $B$ for 180,913 peptides amounting to 2,321 proteins, and in experiment $C$ for 3,699 peptides and 589 proteins.

\section{Question 1) Detection of differentially regulated proteins}

An approach commonly applied to detect differentially regulated proteins is based on the determination of a user-defined threshold in form of a $\mathrm{x}$-fold change in abundance. This method, however, has one significant drawback as it inevitably ignores the different types of variability of a sample. Instead, it is important to find out whether replicate measurements belonging to a protein show a larger variability between different conditions than within the same group [31]. This requires statistical analysis methods such as the one-way analysis of variance (ANOVA). Prior to the application, the highest acceptable significance level $\alpha$ has to be set-common values are 0.05 or 0.01 . Considering a single statistical test one may allow an error of as much as $\alpha$ to falsely reject the null hypothesis. Albeit small for a single test, this error increases dramatically when multiple tests have been performed. This is certainly the case in quantitative proteomics experiments where hundreds to thousands of proteins are investigated in a single experiment. Therefore, this "family-wise error rate" (FWER), which defines the probability that at least one of this type I errors might occur, should be taken into consideration [32-34]. To account for the multiple testing situation all computed $p$-values should be corrected using a method such as proposed by Holm [33]. As already mentioned above, the ANOVA demands certain prerequisites to be fulfilled: i) the assumption that all residues, i. e. deviations from the group's mean, follow a normal distribution can be investigated using a Shapiro-Wilks test [35]; ii) to analyze the homogeneity of variances of each group a Fligner-Killeen test may be utilized [36]. In order to circumvent these requirements, the non-parametric Kruskal-Wallis rank sum test (KW) may be employed as alternative to an ANOVA.

In the present work, we want to determine if both methods detect the same proteins as significantly differentially regulated. In view of the limited number of biological replicates for all three experiments, statistical tests were performed on every peptide measurement, i. e. each abundance ratio determined by a ${ }^{15} \mathrm{~N}$-labeled/ unlabeled peptide pair was considered as an independent measurement of the protein's quantity. If $\mathbf{x}=\left\{x_{i}\right.$, $i=1, \ldots, N\}$ is a series of calculated relative abundance values for a specific protein, and $\mathbf{t}$ denotes an equallysized vector which assigns each value $x_{i}$ a fixed time point $t_{i}$, the (fixed effects) model for the two experiment $A$ and $B$ can be defined as follows:

$$
y=\mathbf{x} \sim \mathbf{t}
$$

In the third experiment $\mathrm{C}$, instead of time the factor carbon source c applies, and each value $x_{i}$ is assigned either the condition benzoate $c_{1}$ or glucose $c_{2}$. This leads to the following model:

$$
y=\mathbf{x} \sim \mathbf{c}
$$

\section{Evaluation of statistical tests}

The acceptable significance level $\alpha$ was set to 0.05 , and all computed $p$-values where corrected by Holm's method. For experiment A, the ANOVA revealed 73 proteins being significantly differentially regulated regarding the five time points (see Table 1 and Additional file 1). However, the Fligner-Killeen test (ii) indicated that 15 of these proteins have inhomogeneous variances. Using the Shapiro-Wilks test (i), moreover, in 29 cases the normal distribution assumption was violated. Taking this into account, strictly speaking, only 38 proteins can therefore be regarded as significantly differentially regulated. The Kruskal-Wallis rank sum test found 64 proteins with significant change in their abundance (see Table 2). In comparison, from the 38 proteins that fulfilled the strict requirements of the ANOVA, 21 were not found significantly regulated by the Kruskal-Wallis test. However, ignoring the strict requirements of normally distributed residues and homogeneous variances, more than $80 \%$ of the proteins (52) that were declared significant by the ANOVA were likewise assessed by the Kruskal-Wallis test. 
Table 1 Significantly expressed proteins - ANOVA result

\begin{tabular}{|c|c|c|c|c|c|}
\hline Accession & $\begin{array}{c}\text { ANOVA } \\
\text { adjusted } p \text {-values }\end{array}$ & $\begin{array}{c}\text { Fligner-Killeen } \\
\text { adjusted } p \text {-values }\end{array}$ & $\begin{array}{c}\text { Shapiro-Wilks } \\
\text { adjusted p-values }\end{array}$ & $\begin{array}{c}\text { Kruskal-Wallis } \\
\text { adjusted p-values }\end{array}$ & $\begin{array}{c}\text { Number of identified } \\
\text { peptide hits }\end{array}$ \\
\hline P40780 & $<0.000001$ & $>0.99$ & $>0.99$ & $<0.000001$ & 741 \\
\hline O34833 & $<0.000001$ & 0.007364 & $<0.000001$ & 0.000001 & 827 \\
\hline O32076 & $<0.000001$ & 0.000083 & $>0.99$ & $<0.000001$ & 1344 \\
\hline O34538 & $<0.000001$ & 0.000020 & 0.977644 & $<0.000001$ & 1616 \\
\hline P94565 & $<0.000001$ & $>0.99$ & 0.264667 & 0.000003 & 1033 \\
\hline P54466 & $<0.000001$ & 0.003793 & $<0.000001$ & $<0.000001$ & 2382 \\
\hline P33166 & $<0.000001$ & $<0.000001$ & 0.067524 & $<0.000001$ & 1605 \\
\hline P02968 & $<0.000001$ & 0.000001 & $<0.000001$ & $<0.000001$ & 9369 \\
\hline P37809 & $<0.000001$ & 0.000002 & 0.007523 & $<0.000001$ & 1112 \\
\hline P46920 & $<0.000001$ & $>0.99$ & $<0.000001$ & $<0.000001$ & 1149 \\
\hline P09124 & $<0.000001$ & $>0.99$ & $>0.99$ & $<0.000001$ & 685 \\
\hline P35136 & $<0.000001$ & $>0.99$ & 0.000003 & $<0.000001$ & 1498 \\
\hline P37808 & $<0.000001$ & $>0.99$ & $>0.99$ & $<0.000001$ & 1219 \\
\hline P94356 & $<0.000001$ & 0.037934 & 0.414572 & $<0.000001$ & 2053 \\
\hline P80877 & $<0.000001$ & $>0.99$ & $>0.99$ & 0.000002 & 892 \\
\hline P27206 & $<0.000001$ & 0.000004 & 0.000028 & $<0.000001$ & 2232 \\
\hline P37871 & $<0.000001$ & 0.399160 & 0.901907 & $<0.000001$ & 1938 \\
\hline O34992 & $<0.000001$ & 0.085647 & $<0.000001$ & $<0.000001$ & 1215 \\
\hline P37476 & $<0.000001$ & $>0.99$ & $<0.000001$ & $<0.000001$ & 3203 \\
\hline Q04747 & $<0.000001$ & $<0.000001$ & 0.000199 & $<0.000001$ & 3460 \\
\hline 031709 & $<0.000001$ & $>0.99$ & $>0.99$ & 0.000020 & 295 \\
\hline P35149 & $<0.000001$ & 0.000169 & $>0.99$ & 0.000693 & 751 \\
\hline P08065 & $<0.000001$ & 0.328408 & $<0.000001$ & $<0.000001$ & 2140 \\
\hline P19582 & $<0.000001$ & $>0.99$ & 0.011528 & 0.000008 & 903 \\
\hline O34966 & $<0.000001$ & 0.012613 & 0.000188 & $<0.000001$ & 1837 \\
\hline P08750 & $<0.000001$ & $>0.99$ & $<0.000001$ & $<0.000001$ & 1109 \\
\hline O34442 & $<0.000001$ & $>0.99$ & 0.047638 & 0.000552 & 291 \\
\hline P24141 & 0.000001 & $>0.99$ & $<0.000001$ & $<0.000001$ & 1257 \\
\hline P25994 & 0.000001 & 0.755894 & 0.006728 & 0.000072 & 1641 \\
\hline 032243 & 0.000003 & 0.599332 & $>0.99$ & 0.000505 & 404 \\
\hline 006745 & 0.000005 & $>0.99$ & 0.013199 & 0.011193 & 485 \\
\hline P37527 & 0.000013 & $>0.99$ & $>0.99$ & 0.051621 & 181 \\
\hline P04969 & 0.000016 & $>0.99$ & $>0.99$ & 0.708837 & 745 \\
\hline O32167 & 0.000020 & $>0.99$ & 0.001374 & 0.000021 & 2891 \\
\hline O32157 & 0.000035 & 0.016583 & $<0.000001$ & 0.000796 & 957 \\
\hline O31501 & 0.000036 & 0.006965 & $<0.000001$ & $<0.000001$ & 1681 \\
\hline P20166 & 0.000046 & 0.272716 & 0.044373 & 0.000003 & 1948 \\
\hline P21471 & 0.000052 & $>0.99$ & $>0.99$ & 0.558509 & 130 \\
\hline P26906 & 0.000063 & $>0.99$ & $>0.99$ & 0.875231 & 300 \\
\hline P54535 & 0.000073 & $>0.99$ & $<0.000001$ & $<0.000001$ & 2391 \\
\hline 031663 & 0.000143 & $>0.99$ & $>0.99$ & 0.378199 & 340 \\
\hline 034633 & 0.000185 & $>0.99$ & $>0.99$ & 0.025892 & 433 \\
\hline P09339 & 0.000203 & 0.244699 & $>0.99$ & 0.040871 & 619 \\
\hline 007516 & 0.000230 & $>0.99$ & $>0.99$ & 0.955961 & 370 \\
\hline P24327 & 0.000234 & $>0.99$ & $<0.000001$ & $<0.000001$ & 1692 \\
\hline P37494 & 0.000244 & $>0.99$ & $>0.99$ & 0.932235 & 241 \\
\hline
\end{tabular}


Table 1 Significantly expressed proteins - ANOVA result (Continued)

\begin{tabular}{|c|c|c|c|c|c|}
\hline P32399 & 0.000267 & $>0.99$ & $>0.99$ & 0.045315 & 388 \\
\hline P39912 & 0.000327 & $>0.99$ & $>0.99$ & 0.002252 & 878 \\
\hline P42971 & 0.000473 & $>0.99$ & $>0.99$ & 0.032372 & 475 \\
\hline P21472 & 0.000594 & $>0.99$ & $>0.99$ & i 0.99 & 357 \\
\hline P21464 & 0.000613 & $>0.99$ & $<0.000001$ & 0.000473 & 1272 \\
\hline P71021 & 0.000703 & $>0.99$ & $>0.99$ & $i 0.99$ & 173 \\
\hline O31567 & 0.001315 & $>0.99$ & 0.000289 & 0.002616 & 613 \\
\hline 006491 & 0.001750 & $>0.99$ & $>0.99$ & 0.542278 & 268 \\
\hline 034789 & 0.001860 & $>0.99$ & $>0.99$ & i 0.99 & 106 \\
\hline 035007 & 0.002474 & $>0.99$ & $>0.99$ & 0.011411 & 410 \\
\hline P71044 & 0.002703 & $>0.99$ & 0.000005 & 0.000284 & 635 \\
\hline P12042 & 0.002703 & $>0.99$ & $>0.99$ & 0.043005 & 699 \\
\hline P46921 & 0.003377 & $>0.99$ & $>0.99$ & 0.421630 & 173 \\
\hline P28611 & 0.004644 & $>0.99$ & $>0.99$ & 0.193988 & 259 \\
\hline P13484 & 0.006385 & $>0.99$ & $>0.99$ & i 0.99 & 208 \\
\hline P39215 & 0.006851 & 0.484875 & $>0.99$ & 0.275825 & 608 \\
\hline 032218 & 0.009165 & $>0.99$ & $>0.99$ & 0.018027 & 567 \\
\hline P18185 & 0.011352 & $>0.99$ & $>0.99$ & $i 0.99$ & 220 \\
\hline P21467 & 0.015346 & $>0.99$ & 0.000001 & 0.001040 & 781 \\
\hline P37870 & 0.017921 & 0.000748 & $>0.99$ & 0.041718 & 1113 \\
\hline 031657 & 0.018006 & $>0.99$ & $>0.99$ & 0.111378 & 227 \\
\hline 006478 & 0.022471 & $>0.99$ & $>0.99$ & 0.859843 & 229 \\
\hline 034878 & 0.022750 & $>0.99$ & $>0.99$ & i 0.99 & 83 \\
\hline 032247 & 0.025031 & $>0.99$ & $>0.99$ & 0.028009 & 845 \\
\hline P39694 & 0.031255 & $>0.99$ & 0.293920 & i 0.99 & 96 \\
\hline P42319 & 0.038119 & $>0.99$ & $>0.99$ & i 0.99 & 224 \\
\hline P49785 & 0.039250 & $>0.99$ & 0.000245 & 0.002557 & 536 \\
\hline
\end{tabular}

Exemplary shown for experiment A, this table displays all ANOVA-reported significantly expressed proteins having a $p$-value below the acceptable significance level of $p<0.05$ (further results can be found in the supplementary data). Proteins are sorted by the ANOVA's $p$-value in ascending order. The assumption that all values are derived from a normal distribution was investigated using a Shapiro-Wilks test: in 29 cases the normal distribution assumption was violated. To analyze the homogeneity of variances of each group a Fligner-Killeen test was performed. Here, 15 of the proteins showed inhomogeneous variances. In summary, only 38 proteins fulfilled all criteria (in bold print). Interestingly, 21 of these proteins were not found significantly regulated by a Kruskal-Wallis test (in italics). All computed $p$-values where corrected using the method described by Holm [33].

For experiment B a performed ANOVA identifies 386 proteins as significantly differently regulated with regard to the factor time (see Additional file 2). While a Fligner-Killeen test (ii) states that 30 of these proteins have inhomogeneous variances, in an impressive number of cases (325 proteins) a violation of the normal distribution assumption was indicated by the Shapiro-Wilks test (i). In summary, only 61 proteins fulfilled the prerequisites of the ANOVA and can therefore-without hesitation-be declared as significantly differentially regulated. Applying in contrast the nonparametric Kruskal-Wallis rank sum test, even 493 proteins reveal significant changes in their abundance between the five time points. Neglecting the requirements of the ANOVA, the agreement between both approaches is higher than $90 \%$ and counts 355 differentially regulated proteins.
In the third experiment $\mathrm{C}$, a comparably small number of only 17 proteins was declared significant by the ANOVA (see Additional file 3). Here, no protein showed any inhomogeneous variances (ii), and only in one case the normal distribution assumption was violated (i). The null hypothesis of no differential regulation was rejected for 10 proteins by the Kruskal-Wallis test, which without any exception were also in the result set of the ANOVA.

To determine a general measure of conformity, resulting $p$-values of the ANOVA and the Kruskal-Wallis test for all proteins were compared using Spearman's rank correlation coefficient [37]. Here, a value of $r=0.8290125$ for experiment A, $r=0.836562$ for experiment $\mathrm{B}$, and $r=0.7780913$ for experiment $\mathrm{C}$ was calculated. Following Cohen's rating of $r \geq 0.5$ as a strong correlation [38], in summary, for all experiments 
Table 2 Significantly expressed proteins - Kruskal-Wallis result

\begin{tabular}{|c|c|c|c|c|c|}
\hline Accession & $\begin{array}{c}\text { ANOVA } \\
\text { adjusted } p \text {-values }\end{array}$ & $\begin{array}{c}\text { Fligner-Killeen } \\
\text { adjusted } p \text {-values }\end{array}$ & $\begin{array}{c}\text { Shapiro-Wilks } \\
\text { adjusted p-values }\end{array}$ & $\begin{array}{c}\text { Kruskal-Wallis } \\
\text { adjusted } p \text {-values }\end{array}$ & $\begin{array}{c}\text { Number of identified } \\
\text { peptide hits }\end{array}$ \\
\hline P40780 & $<0.000001$ & $>0.99$ & $>0.99$ & $<0.000001$ & 741 \\
\hline P24327 & 0.000234 & $>0.99$ & $<0.000001$ & $<0.000001$ & 1692 \\
\hline 032076 & $<0.000001$ & 0.000083 & $>0.99$ & $<0.000001$ & 1344 \\
\hline P24141 & 0.000001 & $>0.99$ & $<0.000001$ & $<0.000001$ & 1257 \\
\hline 034538 & $<0.000001$ & 0.000020 & 0.977644 & $<0.000001$ & 1616 \\
\hline P54535 & 0.000073 & $>0.99$ & $<0.000001$ & $<0.000001$ & 2391 \\
\hline P54466 & $<0.000001$ & 0.003793 & $<0.000001$ & $<0.000001$ & 2382 \\
\hline P02968 & $<0.000001$ & 0.000001 & $<0.000001$ & $<0.000001$ & 9369 \\
\hline 031501 & 0.000036 & 0.006965 & $<0.000001$ & $<0.000001$ & 1681 \\
\hline P46920 & $<0.000001$ & $>0.99$ & $<0.000001$ & $<0.000001$ & 1149 \\
\hline 034966 & $<0.000001$ & 0.012613 & 0.000188 & $<0.000001$ & 1837 \\
\hline P35136 & $<0.000001$ & $>0.99$ & 0.000003 & $<0.000001$ & 1498 \\
\hline P37808 & $<0.000001$ & $>0.99$ & $>0.99$ & $<0.000001$ & 1219 \\
\hline P94356 & $<0.000001$ & 0.037934 & 0.414572 & $<0.000001$ & 2053 \\
\hline P27206 & $<0.000001$ & 0.000004 & 0.000028 & $<0.000001$ & 2232 \\
\hline P37871 & $<0.000001$ & 0.399160 & 0.901907 & $<0.000001$ & 1938 \\
\hline 034992 & $<0.000001$ & 0.085647 & $<0.000001$ & $<0.000001$ & 1215 \\
\hline P37476 & $<0.000001$ & $>0.99$ & $<0.000001$ & $<0.000001$ & 3203 \\
\hline Q04747 & $<0.000001$ & $<0.000001$ & 0.000199 & $<0.000001$ & 3460 \\
\hline P08750 & $<0.000001$ & $>0.99$ & $<0.000001$ & $<0.000001$ & 1109 \\
\hline P33166 & $<0.000001$ & $<0.000001$ & 0.067524 & $<0.000001$ & 1605 \\
\hline P08065 & $<0.000001$ & 0.328408 & $<0.000001$ & $<0.000001$ & 2140 \\
\hline P09124 & $<0.000001$ & $>0.99$ & $>0.99$ & $<0.000001$ & 685 \\
\hline P37809 & $<0.000001$ & 0.000002 & 0.007523 & $<0.000001$ & 1112 \\
\hline 034833 & $<0.000001$ & 0.007364 & $<0.000001$ & 0.000001 & 827 \\
\hline P80877 & $<0.000001$ & $>0.99$ & $>0.99$ & 0.000002 & 892 \\
\hline P20166 & 0.000046 & 0.272716 & 0.044373 & 0.000003 & 1948 \\
\hline P94565 & $<0.000001$ & $>0.99$ & 0.264667 & 0.000003 & 1033 \\
\hline P19582 & $<0.000001$ & $>0.99$ & 0.011528 & 0.000008 & 903 \\
\hline 031709 & $<0.000001$ & $>0.99$ & $>0.99$ & 0.000020 & 295 \\
\hline 032167 & 0.000020 & $>0.99$ & 0.001374 & 0.000021 & 2891 \\
\hline P25994 & 0.000001 & 0.755894 & 0.006728 & 0.000072 & 1641 \\
\hline P71044 & 0.002703 & $>0.99$ & 0.000005 & 0.000284 & 635 \\
\hline P21464 & 0.000613 & $>0.99$ & $<0.000001$ & 0.000473 & 1272 \\
\hline 032243 & 0.000003 & 0.599332 & $>0.99$ & 0.000505 & 404 \\
\hline 034442 & $<0.000001$ & $>0.99$ & 0.047638 & 0.000552 & 291 \\
\hline P35149 & $<0.000001$ & 0.000169 & $>0.99$ & 0.000693 & 751 \\
\hline 032157 & 0.000035 & 0.016583 & $<0.000001$ & 0.000796 & 957 \\
\hline P21467 & 0.015346 & $>0.99$ & 0.000001 & 0.001040 & 781 \\
\hline P39912 & 0.000327 & $>0.99$ & $>0.99$ & 0.002252 & 878 \\
\hline P23446 & $>0.99$ & $>0.99$ & $<0.000001$ & 0.002510 & 451 \\
\hline P49785 & 0.039250 & $>0.99$ & 0.000245 & 0.002557 & 536 \\
\hline 031567 & 0.001315 & $>0.99$ & 0.000289 & 0.002616 & 613 \\
\hline P46922 & 0.069376 & $>0.99$ & 0.000162 & 0.002782 & 581 \\
\hline Q01625 & 0.190501 & $>0.99$ & $<0.000001$ & 0.003031 & 325 \\
\hline P54537 & $>0.99$ & $>0.99$ & $<0.000001$ & 0.004345 & 471 \\
\hline
\end{tabular}


Table 2 Significantly expressed proteins - Kruskal-Wallis result (Continued)

\begin{tabular}{|c|c|c|c|c|c|}
\hline P71070 & 0.417433 & $>0.99$ & 0.000001 & 0.009605 & 314 \\
\hline Q9KWU4 & 0.280189 & $>0.99$ & 0.000448 & 0.009979 & 807 \\
\hline 006745 & 0.000005 & $>0.99$ & 0.013199 & 0.011193 & 485 \\
\hline 035007 & 0.002474 & $>0.99$ & $>0.99$ & 0.011411 & 410 \\
\hline 005252 & 0.111360 & 0.104107 & 0.601796 & 0.017767 & 835 \\
\hline 032218 & 0.009165 & $>0.99$ & $>0.99$ & 0.018027 & 567 \\
\hline 034633 & 0.000185 & $>0.99$ & $>0.99$ & 0.025892 & 433 \\
\hline P94421 & $>0.99$ & $>0.99$ & $<0.000001$ & 0.027798 & 509 \\
\hline 032247 & 0.025031 & $>0.99$ & $>0.99$ & 0.028009 & 845 \\
\hline P42971 & 0.000473 & $>0.99$ & $>0.99$ & 0.032372 & 475 \\
\hline P23447 & 0.078522 & $>0.99$ & 0.721913 & 0.036485 & 459 \\
\hline P09339 & 0.000203 & 0.244699 & $>0.99$ & 0.040871 & 619 \\
\hline P37870 & 0.017921 & 0.000748 & $>0.99$ & 0.041718 & 1113 \\
\hline 032047 & 0.178170 & $>0.99$ & $<0.000001$ & 0.042466 & 665 \\
\hline P12042 & 0.002703 & $>0.99$ & $>0.99$ & 0.043005 & 699 \\
\hline P32399 & 0.000267 & $>0.99$ & $>0.99$ & 0.045315 & 388 \\
\hline P08066 & 0.105333 & $>0.99$ & 0.000002 & 0.048719 & 644 \\
\hline P40779 & 0.083444 & $>0.99$ & 0.053914 & 0.049061 & 409 \\
\hline
\end{tabular}

Exemplary shown for experiment A, the table displays all significantly expressed proteins ( $p<0.05, p$-value adjustment using Holm) reported by a Kruskal-Wallis rank sum test (further results can be found in the supplementary data). Proteins are sorted by the Kruskal-Wallis' $p$-value in ascending order. In summary, 64 proteins were found with a significant change in their abundance. By comparison, 52 of them were also reported as significant by the ANOVA (not significant printed in italics).

a large degree of similarity between both results can be attested.

\section{Visualization of statistical tests}

A simple but also very powerful way to visualize the results of an ANOVA and review individual proteins, e. g. if statistical significance is doubtable, are box- and whisker plots [39]. These provide an overview of five essential characteristics of a series of measurements to compare distribution and relative location between different groups. Figure 1 contains four plots that visualize the differences between the calculated abundance ratios of four selected proteins over time. As an example, both the ANOVA as well as the Kruskal-Wallis test show a significant change in abundance of the protein P40780 in experiment A. Although the measurements are not following a Gaussian distribution, there is clearly a differential regulation over time. The membrane protein Q01625 reveals only small changes and was regarded significant by the Kruskal-Wallis test, but not by the ANOVA after $p$-value adjustment. P39126, a NADPdependent dehydrogenase, is not showing any clearly distinguishable and significant pattern of expression. A reason therefore might be a high biological variance but, of course, also technical errors in measurement. Fortunately, the same-albeit to an even greater degreeapplies for the human protein $\mathrm{K} 1 \mathrm{C} 10$, which is an obvious contamination.

\section{Question 2) Identification of co-regulated proteins}

Applying cluster analysis on isotope-labeled quantitative proteomics datasets aims to identify proteins that reveal similar patterns of regulation. To this end, the clustering process aggregates those proteins in groups that are characterized by a similar series of measurements. Accordingly, a solution has to be found i) to determine the similarity for two proteins $\mathbf{x}=\left\{x_{i}, i=1, \ldots, N\right\}$ and $\mathbf{y}=\left\{y_{i}, i=1, \ldots, N\right\}$, and ii) to aggregate clusters from these similarity values, i. e. the formulation of an algorithm. These two problems span the space of algorithmic solutions to the clustering problem. While an answer to question 1 was searched on the peptide level, cluster analysis demands averaging over all calculated peptide abundance ratios to form one value per protein and condition. Being one of the most frequently used statistics for this purpose, here the arithmetic mean was selected, though, also the median or the trimmed mean could have been a good choice. Aiming to achieve utmost accurate analysis results, only those proteins where included having at least two peptide measurements per condition. These are 188 proteins for experiment A, 935 for experiment B, and 196 for experiment C. At this point, we intentionally decided against taking into account more proteins in our analysis as this may have resulted in the necessity to replace missing values in the data. For experiment A, this was exemplary 


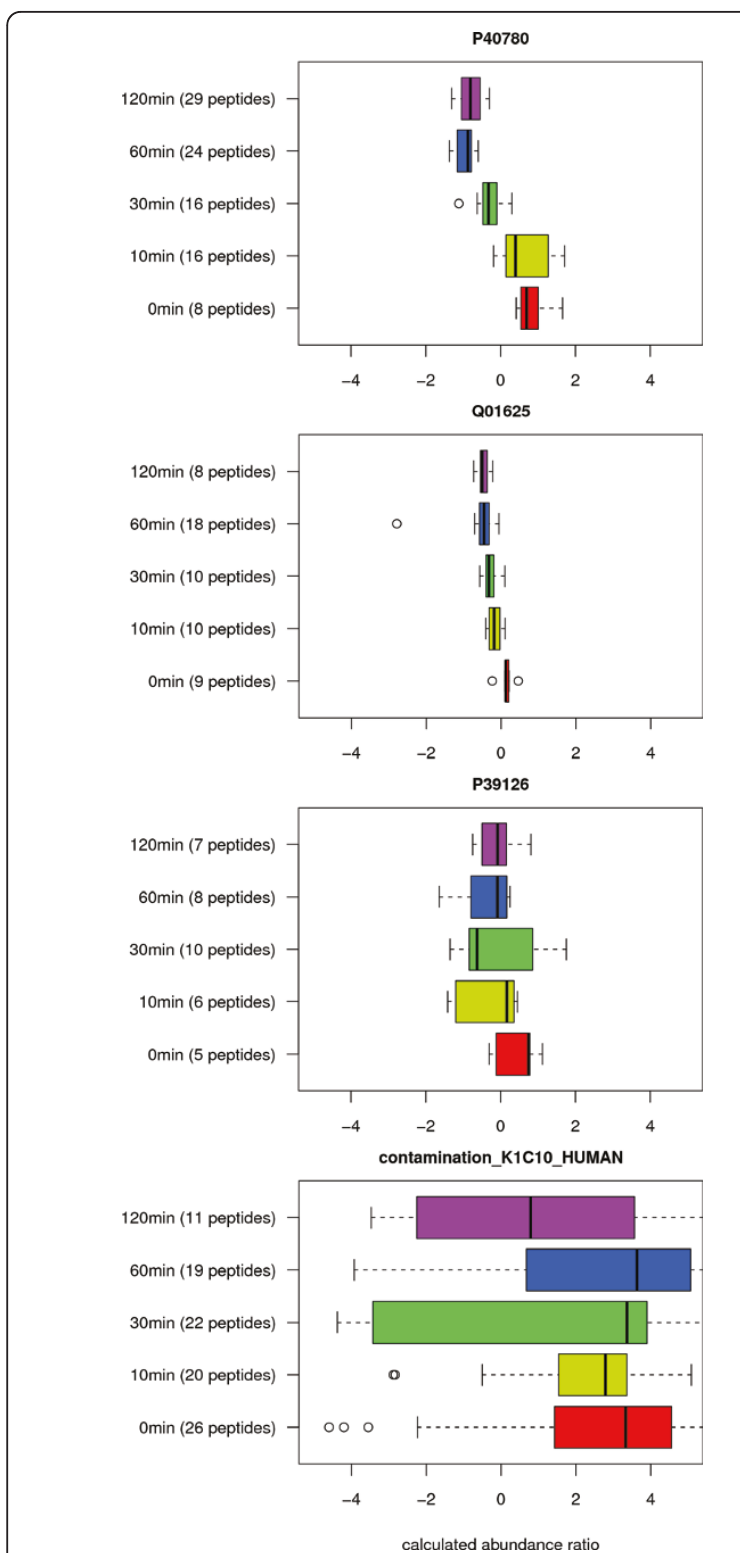

Figure 1 Box-whisker plot. Box- and whisker plots provide a simple but also very powerful way to visualize the results of an ANOVA, and give an overview of five essential characteristics of a series of measurements including median, lower and upper quartiles, and extreme values. This figure demonstrates the differences between the calculated abundance ratios of four proteins. Both the ANOVA as well as the Kruskal-Wallis test show a significant change in abundance of the protein P40780 in experiment A. Although the measurements are not following a Gaussian distribution, there is clearly a differential regulation over time. The membrane protein Q01625 reveals only small changes. It was nonetheless regarded significant by the Kruskal-Wallis test, but not by the ANOVA after $p$-value adjustment. P39126, a NADPdependent dehydrogenase, is not showing any clearly distinguishable and significant pattern of expression. A reason therefore might be a high biological variance but, of course, also technical errors in measurement. Fortunately, the same-albeit to an even greater degree-applies for the human protein $\mathrm{K} 1 \mathrm{C} 10$, which is obviously a contamination. implemented and tested by replacing any missing value with each protein's mean abundance ratio over all conditions. Allowing for example one missing value per protein in the data, cluster analysis would cover 263 proteins for this experiment. Since further analysis showed comparable clustering results (data shown in $\mathrm{QuPE}$ ), we refrained, in the following, from including any protein having less than two measurements per conditions.

Given the matrix of protein ratios per condition, a common solution to the clustering problem i) is to apply the Euclidean distance. This can be interpreted as the physical distance between two points, and is, hence, very appealing [20]. Given $\mathbf{x}, \mathbf{y}$ this distance $d$ is defined as follows:

$$
d(\mathbf{x}, \mathbf{y})=\sqrt{\sum_{i=1}^{n}\left|x_{i}-y_{i}\right|^{2}}
$$

In some cases, actual differences in the abundance ratios of two proteins are negligible but instead a positive or negative correlation between two proteins is of interest. Under these conditions similarity measures based on correlation such as Pearson's uncentered or centered correlation coefficient may be utilized (see Supplementary material). However, it has to be considered that this method may regard two proteins as similar although one is overly up- and one overly downregulated.

The cluster algorithm to solve ii) determines how all measurements, i. e. proteins, are to be grouped into clusters. Two opposing properties to characterize a cluster result are connectedness and compactness [27]. Transfered to the context of proteomics this can be seen as the conflict between the two ideas to, on the one hand, combine as many proteins as possible if they reveal only a slight similarity and to form compact clusters that contain only those proteins that are utmost similar, on the other hand (see Figure 2). Hierarchical cluster analysis (HCA) methods (a) organize the input data (i. e. the measurements) into a tree structure exposing the relationships from the most similar to the most different proteins. Using some straightforward criterion (like a horizontal cut through the tree) clusters are generated from the result. Single- and CompleteLinkage are two approaches that represent the aforementioned opposing properties [21]. Average-Linkage can be regarded a compromise of both approaches, and Ward's method is based on the idea that each time two clusters of proteins are combined the variance within this new cluster will increase-an increase that should be as minimal as possible [22,23,40]. In contrast, partitioning cluster algorithms (b) follow an optimization 


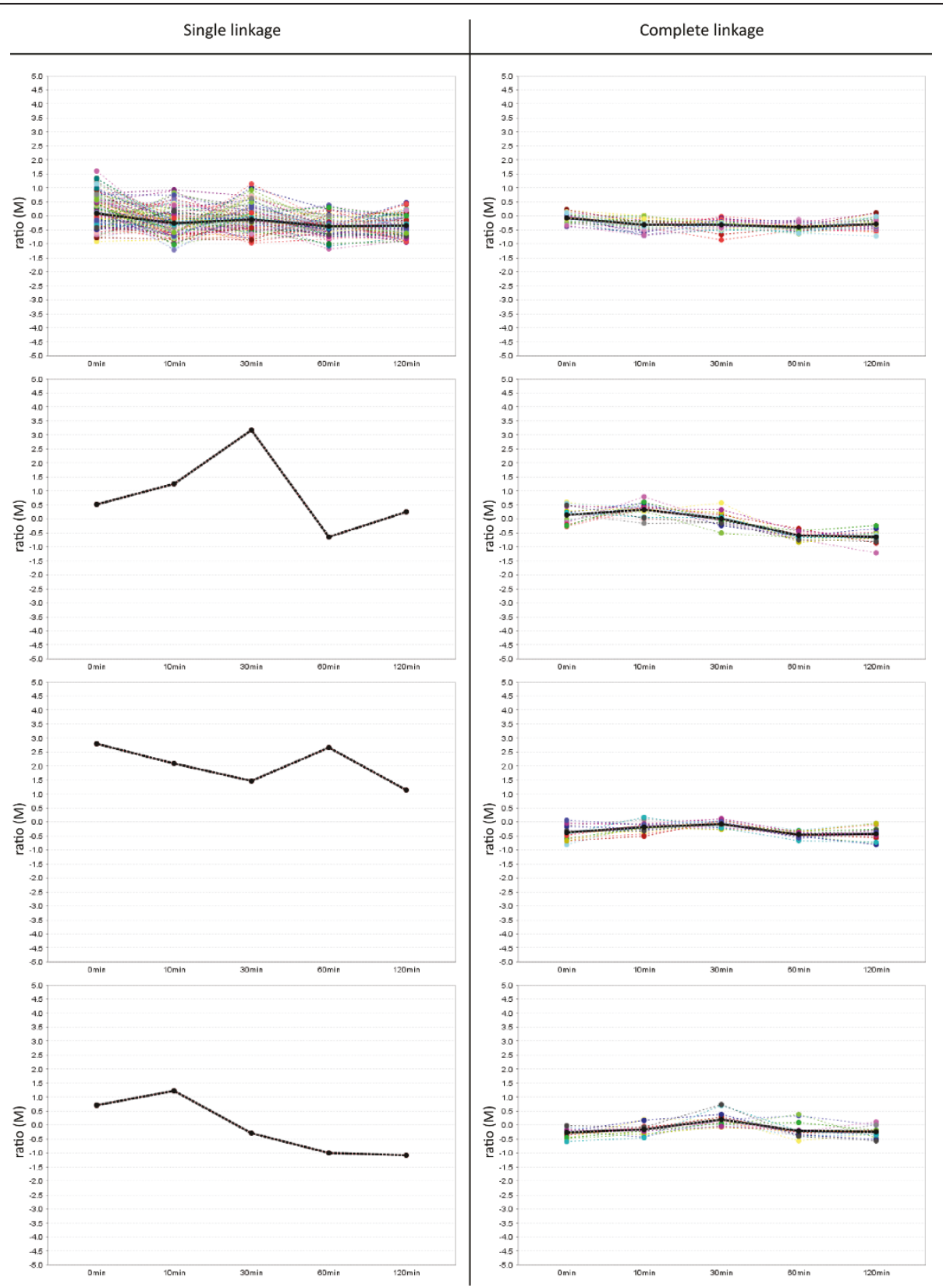

Figure 2 Comparison of cluster properties - connectedness vs. compactness. This figure shows each four selected clusters that resulted from two hierarchical cluster analyses using Single-Linkage (on the left side) and Complete-Linkage (on the right side). These results demonstrate the two opposing properties of a clustering connectedness and compactness: Single-Linkage, on the one hand, tends to group as many proteins in a cluster if there is at least a slight similarity. This often leads to one large cluster that contains most of the proteins while other clusters consist of individual outliers. Complete-Linkage, on the other hand, tries to form compact clusters that contain only those proteins that are utmost similar.

strategy to successively assign each protein of the input dataset to one distinct group. In the outcome, each of these clusters is characterized by a typical representative-its cluster center or profile-allowing for a direct reading of the cluster's mean abundance ratios. The K-means algorithm $[24,25]$, the most prominent member of this group of cluster algorithms, has a clear disadvantage as it strongly depends on the initial definition of these group centers and repeated invocation might therefore yield varying results. Neuralgas claims to be an enhancement as it takes into account a "neighborhood ranking" of all proteins that are assigned to a cluster-an advantage bought by an increase in computational running time [41]. To analyze this problem in 
terms of reproducibility, both K-means and Neuralgas were executed 25 times with a fixed cluster number of 20 . In each repetition, the initial cluster centers were randomly sampled from the input dataset (experiment A). A pairwise degree of similarity between each two clustering results was then computed using the Rand index [42]. Here, a value of $R=0.0$ indicates no similarity, while $R=1.0$ means that the results are identical. In all cases the outcomes of two invocations of both algorithms are slightly dissimilar, ranging from $R=0.89$ to 0.98. In comparison, however, as shown in Figure 3, the K-means approach reveals a significant lower similarity between two results $(p<0.001)$, in other words, a lower reproducibility. Density-based cluster algorithms (c) such as fuzzy C-means [26] allow a fuzzy assignment of each data point/protein to one or more clusters. However, to compare the results to other clusterings, in the end, each protein is assigned to that cluster which it most likely belongs to, i. e. the cluster with maximal membership.

\section{Evaluation of cluster algorithms}

Given this plenitude of algorithmic approaches to solve the clustering problem one may ask in how far their outcomes differ, particularly, applied to quantitative proteomics data. Without any interpretation of the resulting clusterings, we therefore estimated a pairwise degree of similarity between two clustering results both with identical cluster numbers produced by two different algorithms. For this purpose, the adjusted Rand measure [42] was utilized. Figure 4 visualizes the mean of all Rand indexes computed for cluster numbers from two to 50 for experiment $\mathrm{A}$ and $\mathrm{C}$, and from two to 100 for experiment B. For the latter, we selected a different highest cluster number in respect to the experiment's dataset size and, hence, its increased number of quantified proteins. A strong but not surprising degree of similarity (A/B: $R>0.45$, C: $R>0.6$ ) was found between the two methods K-means and Neuralgas. Furthermore, both methods show a comparably high similarity (up to $R>0.6$ in experiment C) to HCA using Ward's linkage and Euclidean distances (Ward/Euclidean); in experiment $\mathrm{C}$, in addition, to fuzzy C-means, Complete- and less pronounced to Average-Linkage (the two latter with Euclidean distances). Only in experiments $\mathrm{A}$ and $\mathrm{C}$, a pronounced similarity $(R>0.45)$ can be attested to the outcomes of HCA using Complete- and AverageLinkage (Complete/Euclidean, Average/Euclidean). In experiments A and B, a slight similarity is, furthermore, found between Single- and Average-Linkage (likewise with Euclidean distances). On the contrary, it has to be pointed out that methods such as Average-Linkage using correlation-based distances or, with the aforementioned exception, Single-Linkage using Euclidean distances each yielded an entirely unique output. In summary, the results of this comparison (see Supplementary information for further details) demonstrate that the choice for a cluster algorithm is not arbitrary but instead strongly influences the outcome.

From a computational point of view, a number of quality measures have been proposed to evaluate and rank the outcomes of cluster algorithms. Because of opposing characteristics such as compactness and connectedness, however, no definite criteria can be formulated that describes an optimal clustering of a dataset. This pertains not only to the applied cluster algorithm but also to the "true" number of clusters of a dataset. Proposed measures that base solely on the clustering itself and the underlying dataset [27] range from early approaches [43-45] up to novel instruments [46,47] (see Additional file 4 for further details). In hierarchical cluster analysis, a simple but powerful way to assess the "true" number of clusters of a dataset is a visual analysis of each possible cluster number set in relation to the distance (similarity) between the two clusters that are merged to gain a clustering of this size. An optimal solution can be identified by searching a knee in the plot (see Figure 5 for an example).

From a biological point of view, a good cluster solution is much more difficult to assess. In general, this demands additional knowledge about the proteins under investigation, e. g. a set of known class labels or a previously determined analysis result. A calculated clustering could then be compared to the labels to determine a degree of similarity. In real life experiments this information is, however, rarely available for all analyzed proteins. An automatic evaluation based on external information is, hence, nearly impossible. Nevertheless, biologically meaningful clusters are characterized by consisting of proteins that belong to a similar functional category or which are involved in the same metabolic pathway.

Assistance in choosing a cluster algorithm, particularly, for the analysis of gene expression data, was recently offered by Yeung et al. [46]. They delineated an instrument called Figure of Merit (FOM) to evaluate cluster solutions. The idea of their method is to integrate a kind of bootstrapping approach (cf. [18]), and thereby to estimate the predictive power of a cluster algorithm. Applied on our data, this index revealed Ward/Euclidean, K-means as well as Neuralgas as the best performing cluster algorithms, while correlationbased cluster algorithms, Single-Linkage using Euclidean distances, and-at least in two experiments-fuzzy C-means produce the least reliable results (see Figure 6).

Aiming to determine an optimal clustering of each proteomics dataset regarding both the biological as well as the computational point of view, we analyzed the results of all applied cluster algorithms using a 

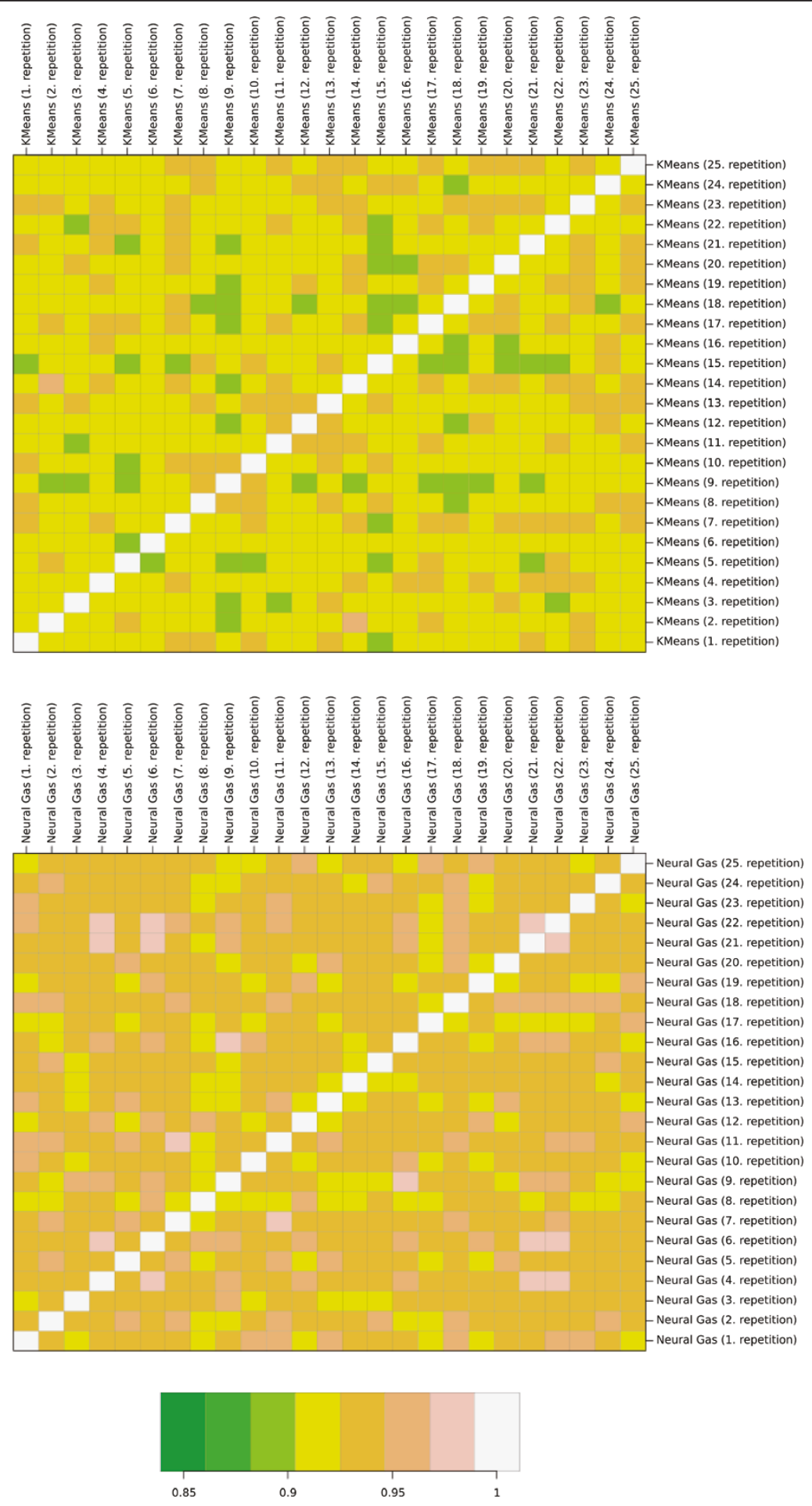

Figure 3 Comparison of K-means and Neuralgas using the Rand measure. This figure visualizes the problems of K-means in terms of reproducibility. Both K-means and Neuralgas were executed 25 times with a fixed cluster number of 20. In each repetition, the initial cluster centers were randomly sampled from the input dataset. A pairwise degree of similarity between each two clustering results was then computed using the Rand index. A value of 0.0 indicates no similarity, while a value of 1.0 means identity. The shown heatmaps visualize this measure. The upper part of this figure displays the results of K-means, the lower part those of Neuralgas. In all cases the outcomes of two invocations of both algorithms are slightly dissimilar, and not identical. However, the K-means approach reveals a significant higher variance in its results. 

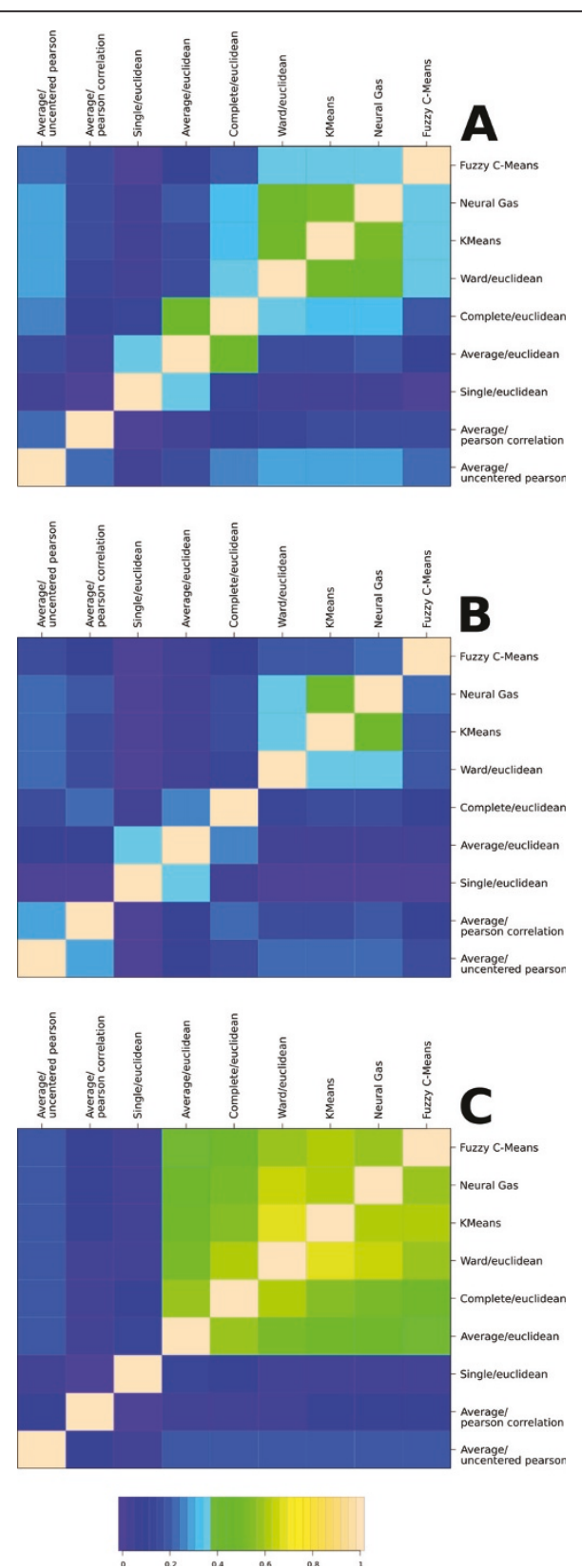

Figure 4 Comparison of cluster algorithms using the Rand measure. We estimated a pairwise degree of similarity between two clustering results both with identical cluster numbers produced by two different algorithms using the adjusted Rand index. The three heatmaps shown in this figure each visualize the mean of all Rand indexes computed for cluster numbers from two to 50 for experiment A and C. For experiment B, we selected a different highest cluster number-here 100 was chosen-in respect to the experiment's dataset size and, hence, its higher number of quantified proteins. In all three experiments, notably, a high degree of similarity is observed between the three cluster methods K-means, Neuralgas, and HCA using Ward's Linkage; in experiment $\mathrm{C}$, in addition, to fuzzy C-means, Complete- and less pronounced to Average-Linkage (the two latter with Euclidean distances). In experiments A and B, a slight similarity is, furthermore, found between Single- and AverageLinkage (likewise with Euclidean distances). diversified selection of cluster indexes. Here, the index of Calinski and Harabasz [43], which sets the similarity of all proteins grouping together in a cluster in relation to the dissimilarities of each two clusters, and even more the Index I [47], which follows a comparable approach, tend to favor smaller cluster numbers between two and three clusters (see Figures 7 and Figure 8; Additional files 5, 6 and 7 for further details). While from a computational point of view these results seem reasonable, from a biological point of view they do not allow any meaningful interpretation of the data. In general, these small clusterings only characterize individual outliers, while the rest of the clusters are found with a high number of cluster members having everything clustered together that reveals only a slight similarity. Experiment $\mathrm{C}$ is, in some respect, an exception as here the cluster index of Calinski and Harabasz gives evidence for higher cluster numbers, e. g. 14 for Complete/Euclidean. This could result from the fact that the data of this experiment has a comparably low dimensionality as there are only two different abundance ratios per protein-one for growth on benzoate, one for glucose.

Davies and Bouldin formulated a general framework for the evaluation of the outcomes of cluster algorithms [44]. An instance of their index provided by Halkidi et. al [28] follows the idea that an optimal solution to the clustering problem has been found as soon as for each cluster no other utmost similar cluster-with regard to the intra-cluster error sum of squares as well as the distance between clusters-can be identified. In contrast to other indexes, this is indicated by the minimal calculated index value (see Figure 9). In experiment A, for instance, for the two cluster algorithms K-means and Neuralgas, a local minimum can be located around the 30 -cluster solution. A general interpretation of this index, however, seems to be difficult due to a strong tendency towards constantly decreasing index values with regard to large cluster numbers. An exception are both correlation-based cluster algorithms (Average/Pearson correlation, Average/Uncentered Pearson): at least for experiment $C$, index values seem constantly to increase providing nevertheless no clear statement with regard to an optimal clustering of the data.

We draw conclusions differing from that obtained in a microarray study [48], when we investigated the index of Krzanowski and Lai [45]. In that study-a comparison of five cluster measures on six different microarray datasets-the index revealed a poor performance in terms of predictive power. However, in our analysis the application showed both from a biological as well as from a computational point of view meaningful results (see Figure 10). For our proteomics dataset of experiment $A$, the index suggested a cluster number between 


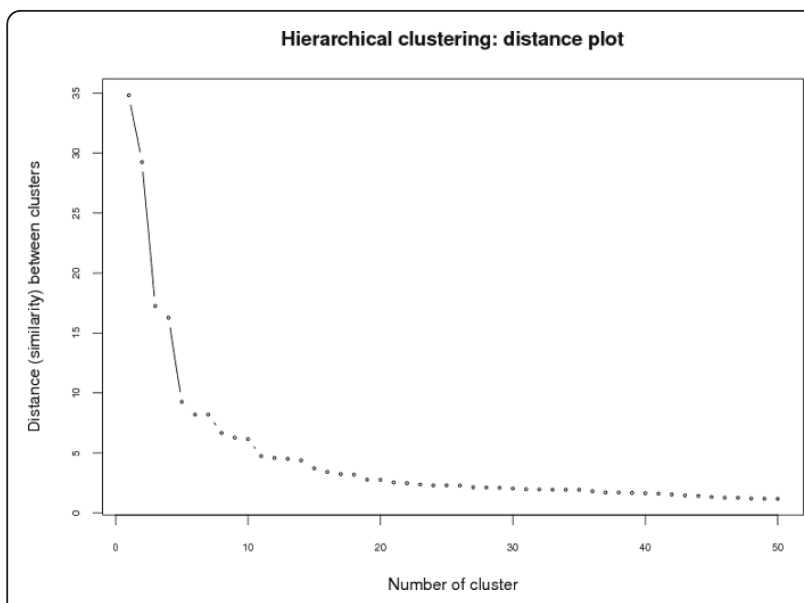

Figure 5 HCA - distance plot. In hierarchical cluster analysis, a simple but powerful way to assess the "true" number of clusters of a dataset is a visual analysis of each possible cluster number set in relation to the distance (similarity) between two clusters that will be merged. An optimal solution can be identified by searching a "knee" in the plot. In this example this can be identified around the 10-cluster solution.

three (Ward/Euclidean), which also shows a local maximum at 23 clusters, and 43 clusters (Average/ Uncentered Pearson). To extend our knowledge about the identified proteins, information from COG (clusters of orthologous groups of proteins) [49] and the Kyoto Encyclopedia of Genes and Genomes (KEGG) [50] was integrated. Looking at the 23-cluster solution produced by Ward/Euclidean in detail, the outcome reveals a reasonable biological finding. It consists of several clusters of proteins sharing a common function, e. g. regarding cell wall biogenesis, metabolism of amino acids, or motility and chemotaxis, and corresponds to the findings of Hahne et al. [1]. Proteins that reveal a similar pattern of regulation are for example eight proteins that are involved in amino acid transport and metabolism. The proteins in this cluster appeared down-regulated after 30 minutes. In another cluster eight proteins, which are mostly responsible for cell motility, show an increase in their relative abundance over time.

For experiment B, the index of Krzanowski and Lai displays cluster numbers between 14 (Average/Pearson correlation) and 70 (Complete/Euclidean), whereby, inter alia, a 43-cluster solution for HCA using Ward and Euclidean distances sparked our interests. This solution distinguishes several groups of proteins according to their different regulation during the time course. Analogous to the results of Otto et al. [2] a number of proteins were found with decreasing abundance ratios after cells entered stationary phase. These are, presumably, subjected to degradation. The resulting clustering included a group of 31 proteins, which

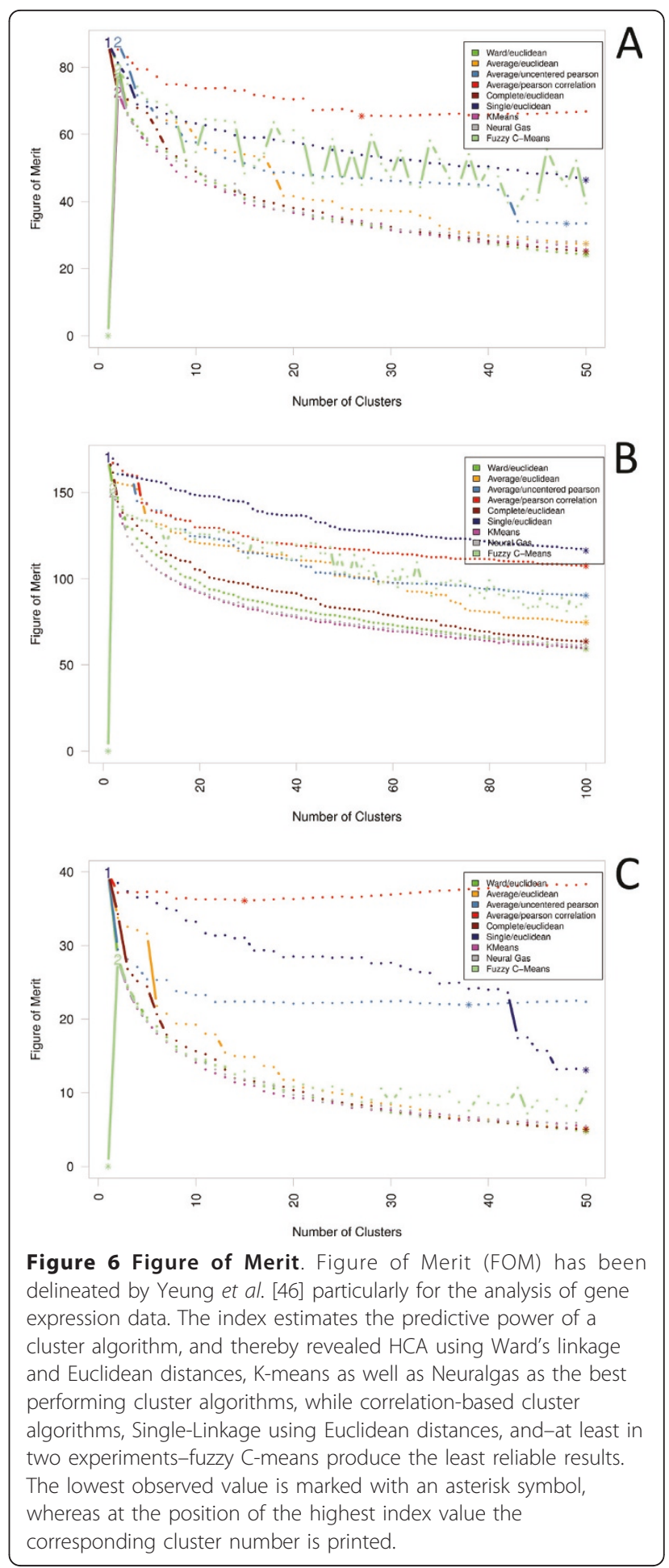

play a role in the metabolism of nucleotides and amino acids; a cluster of 10 proteins similarly involved in secondary metabolites biosynthesis, transport and catabolism; and another cluster with 20 functionally related proteins with regard to amino acid transport and metabolism. On the opposite, a cluster could be 

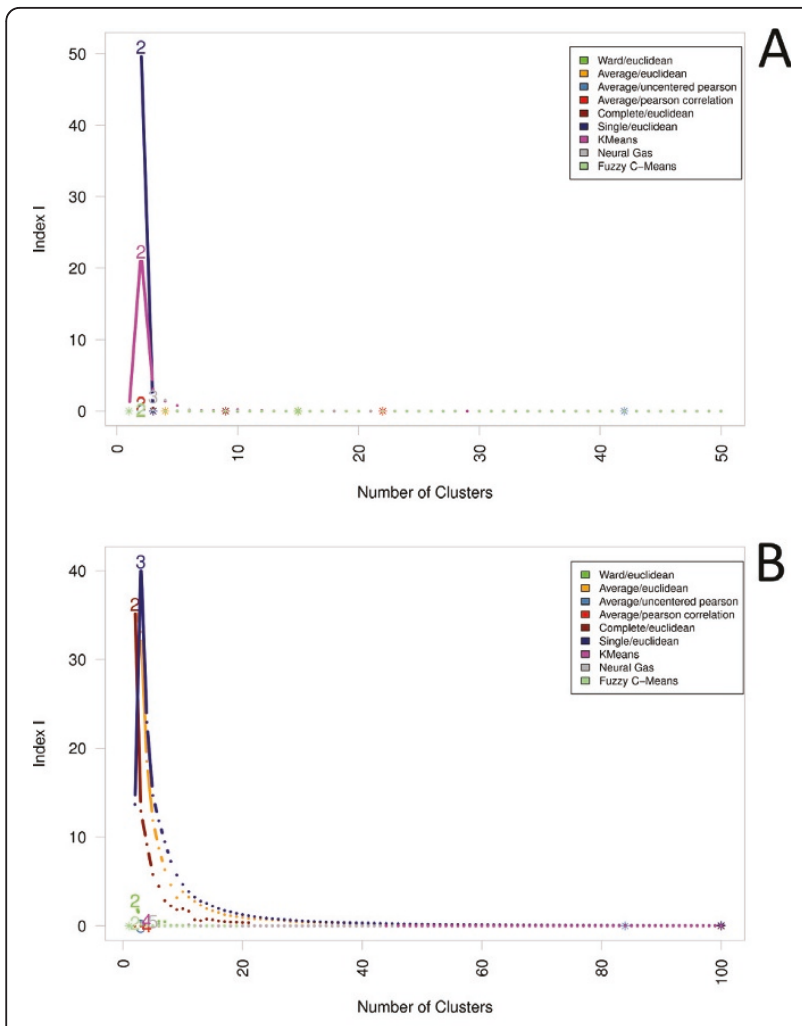

B

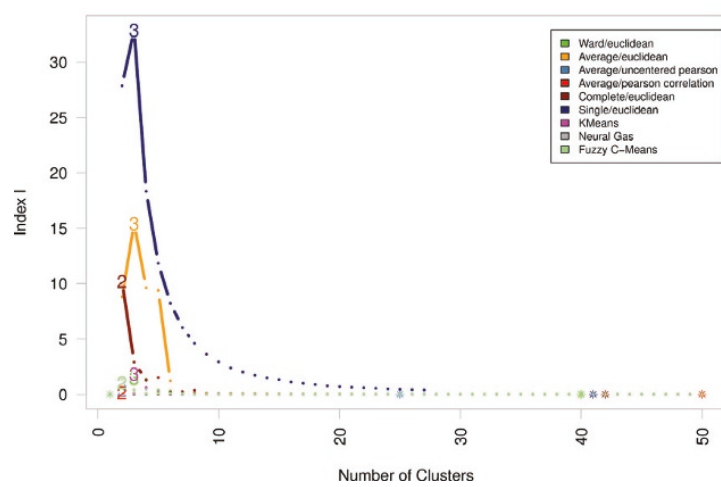

Figure $\mathbf{7}$ Index I. The cluster index "Index I" tends to favor smaller cluster numbers between two and three clusters. From a computational point of view this is clearly a good result. Unfortunately, from a biological point this does not allow any meaningful interpretation of the data. In general, these small clusterings only characterize individual outliers, while the rest of the clusters are found with a high number of cluster members having everything clustered together that reveals only a slight similarity.

identified with 10 proteins strongly increasing in amount after the transition from exponential to stationary growth phase. Specifically, these are, for example, the proteins $\mathrm{O} 34425$ and P54418, which were also highlighted as significantly differentially regulated in the original publication.

Meaningful results where also observed in the application of the index of Krzanowski and Lai on the data
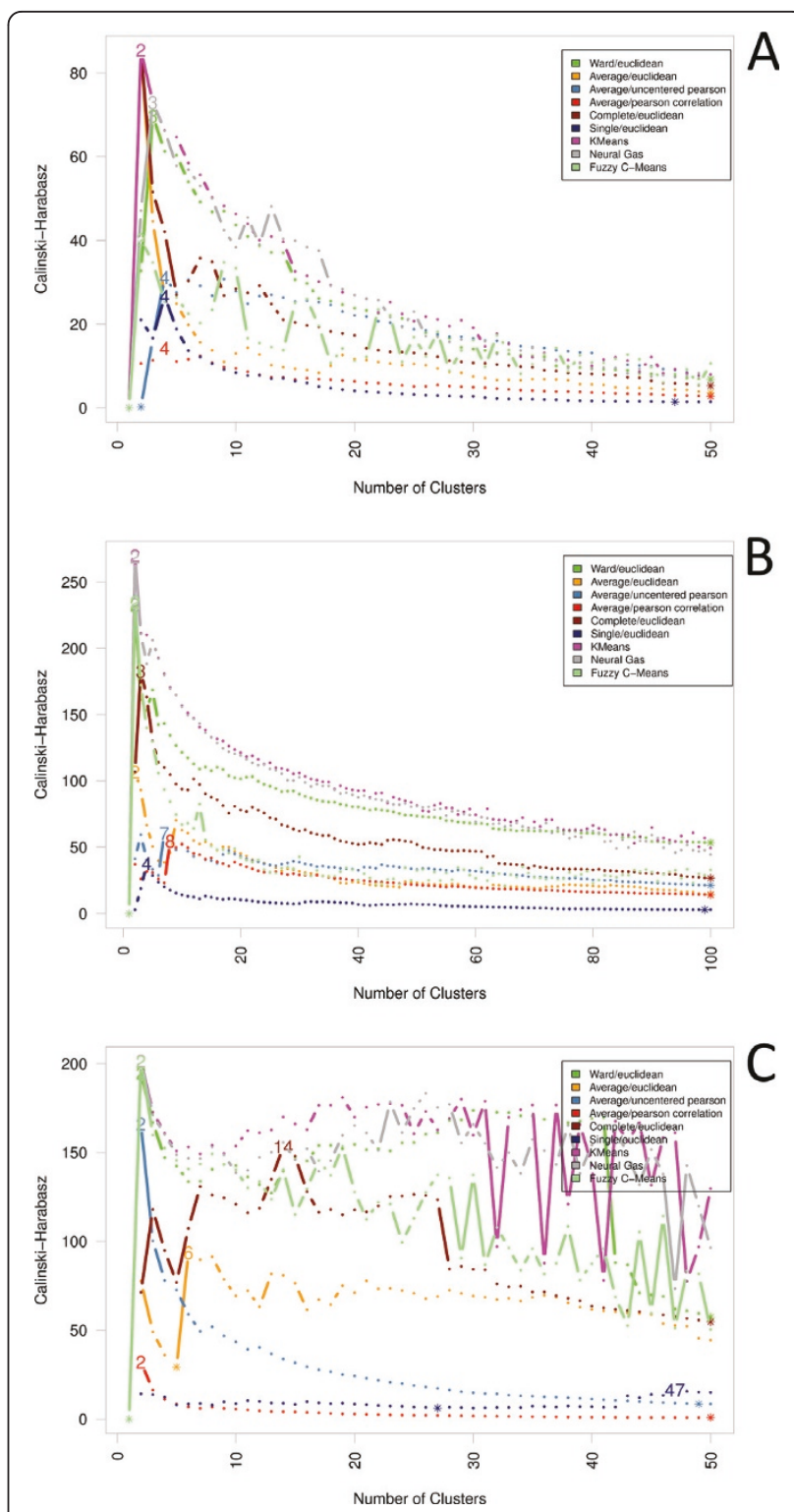

Figure $\mathbf{8}$ Calinski-Harabasz. Similar to the "Index I" the cluster index of Calinski and Harabasz tends to favor smaller cluster numbers between three and four clusters. In the same manner, the applicability with respect to the biological question also remains questionable.

of experiment C. Here, an optimal clustering was found for example at seven clusters for Average/ Uncentered Pearson, 22 clusters for Ward/Euclidean and 38 clusters for Average/Euclidean. In the 22-cluster solution using Ward/Euclidean a number of ribosomal proteins showed no change in regulation due to the two different growth media. In contrast, proteins belonging to the COG functional categories amino acid transport and metabolism, and energy production were down-regulated during growth on benzoate 


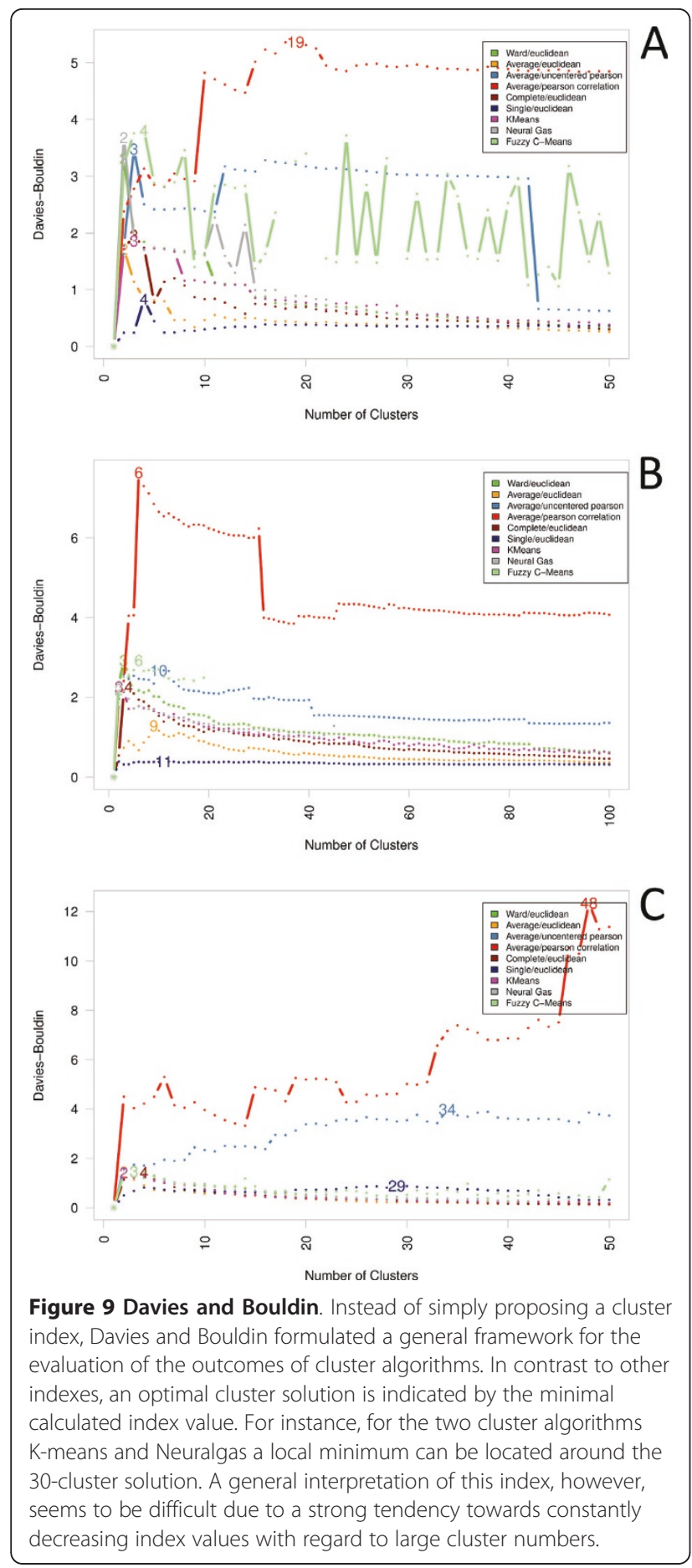

including for example Cg1806, an enzyme involved in sulfur metabolism.

\section{Visualization of cluster results}

A typical visualization of the results of a hierarchical cluster analysis is a heatmap as exemplary shown in Figure 11[51]. Calculated abundance ratios are color coded. An attached dendrogram reveals the

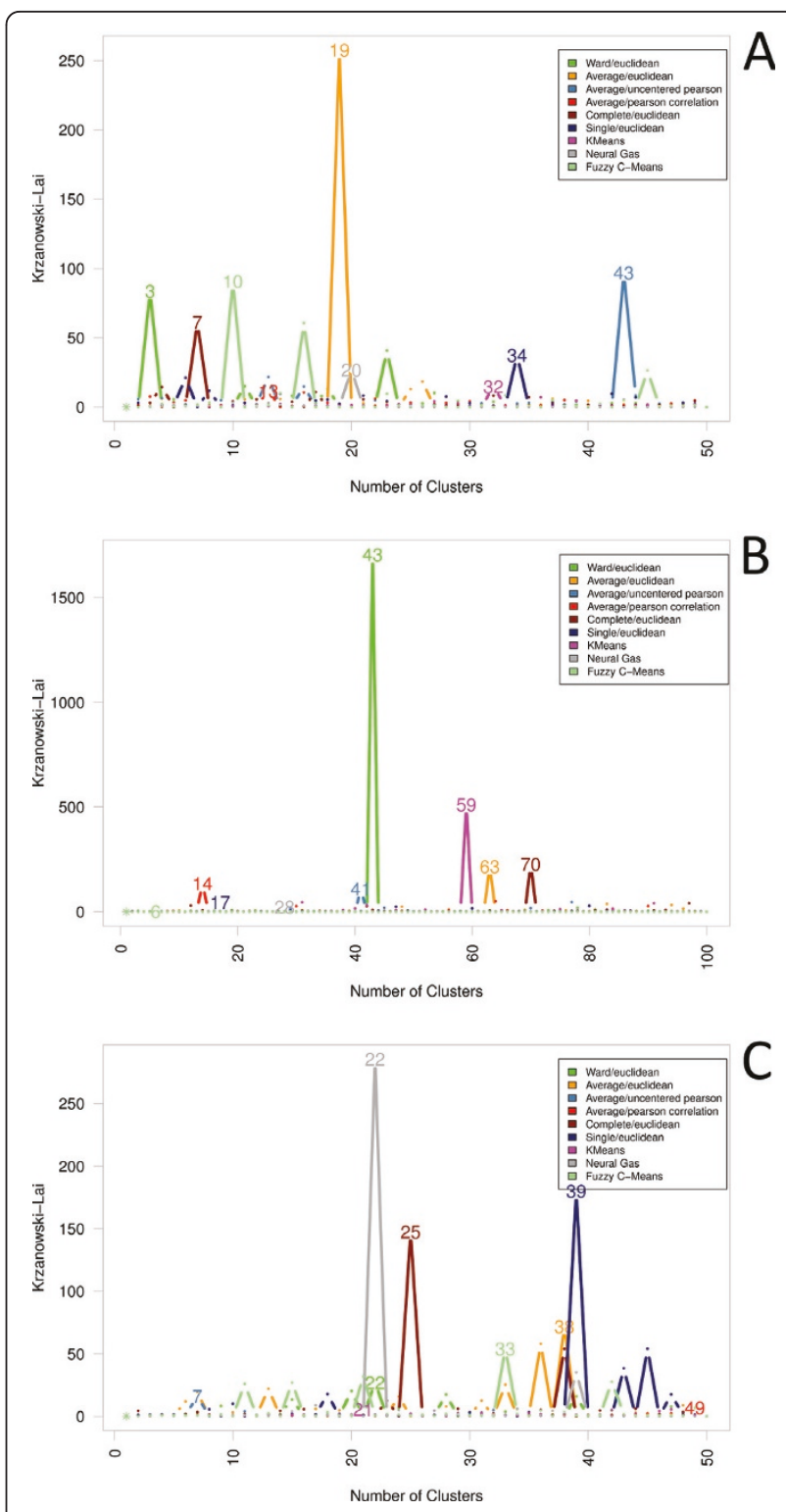

Figure 10 Krzanowski and Lai. The cluster index of Krzanowski and Lai showed both from a biological as well as from a computational point of view meaningful cluster numbers: for the data of experiment $A$, there were found cluster numbers between 3 for Ward/Euclidean-here a second local maximum was found at 23 clusters-and 43 for Average/Uncentered Pearson as the true clustering of the data; for experiment B, between 14 (Average/ Pearson correlation) and 70 (Complete/Euclidean), with a protruding 43-cluster solution applying Ward/Euclidean; and for experiment C, e. g. at seven clusters for Average/Uncentered Pearson, 22 for Ward/ Euclidean and 38 for Average/Euclidean.

hierarchical relations between the proteins. In many cases, one is not interested in determining the relationships between all proteins, but instead of representative groups of proteins that show a very similar pattern of regulation. Here, a simple XY-plot may provide an adequate visualization. 


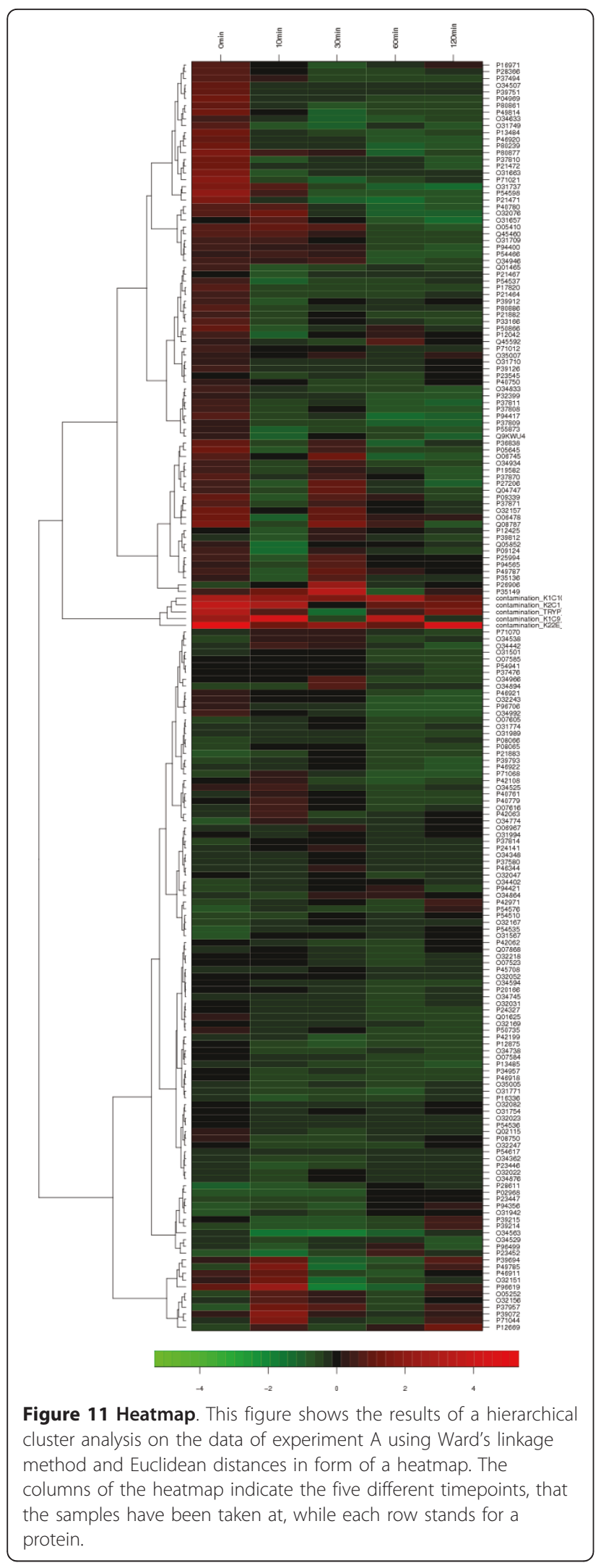

\section{Conclusions}

This work aims at paving a straight path through the jungle of computational methods to analyze mass spectrometry-based isotope-labeled datasets, targeting the two questions that typically arise in proteomics experiments: 1) which proteins are differentially regulated regarding the selected experimental conditions, and 2) are there groups of proteins that show similar abundance ratios, indicating that they have a similar turnover? In contrast to other types of Omics experiments, mass spectrometry-based proteomics is faced with particular challenges: due to background signals in mass spectra the data is for example comparatively noisy, and, because of unidentified peptides, values are missing from the measurements [52]. To take these problems into account, we developed our evaluation strategy based on three recently published datasets on Bacillus subtilis and Corynebacterium glutamicum. In an ideal situation, we would expect that two commonly applied tests to answer question one reveal the same proteins as significantly differentially regulated, and indeed, there was found a strong congruence between the outcomes of an ANOVA and a Kruskal-Wallis rank sum test. However, an ANOVA, strictly speaking, in many cases could not be evaluated, because the normal distribution assumption was often not fulfilled. "Asking whether ANOVA [...] assumptions are satisfied is not idle curiosity. The assumptions of most mathematical models are always false to a greater or lesser extent. The relevant question is not whether ANOVA assumptions are met exactly, but rather whether the plausible violations of the assumptions have serious consequences on the validity of probability statements based on the standard assumptions." [[53], p.237]. As an example, differences in the abundance ratios of the protein P40780 (experiment A, see Figure 1) suggest that, in this case, the normal distribution assumption may be negligible. In conclusion, we recommend to firstly rely on the results of an ANOVA, but secondly, to always take into consideration Kruskal-Wallis. Results should then be compared and further visually investigated using for example Box- and Whisker-plots. In all tests, because of the multiple testing situation, adjustment of computed $p$-value should take place.

Question two is even harder to answer: With the aim of producing biologically meaningful results, we are clearly interested in grouping those proteins in a cluster that reveal an utmost similar pattern of abundance ratios in our experiment. Hence, Single-linkage is not applicable for this purpose, which is also proven by the development of the Figure of Merit. If the benefits of a hierarchical cluster analysis are requested, Ward's 
method has proven a good choice. If there isn't, Neuralgas should be selected, which clearly outperforms the Kmeans approach, in particular, regarding the reproducibility of its results. The only drawback of this algorithm might be its comparatively high computational complexity, which is, however, negligible taken into consideration today's average computing resources. In our application study, we found-from a biological point of view-interesting clusters of proteins that both revealed a similar pattern of regulation and fulfilled a similar biological function using these two approaches.

Correlation-based distance measures should only be applied if they can be justified by the underlying experimental hypotheses, e. g. if proteins are expected to be commonly regulated but not at an equal level of abundance. The most difficult part is the validation of a cluster result to gain the "true" number of clusters of a dataset. Here, the cluster index of Krzanowski and Lai turned out to produce both computationally as well as biologically meaningful results. In contrast to other investigated validity measures the index solely relies on the internal compactness of clusters, which seems to correspond to our objective of clustering those proteins that reveal a highly similar pattern of regulation.

To further evaluate cluster analysis results, we recommend including annotation data, such as functional categories. If for example a cluster analysis reveals a group of proteins similarly regulated that furthermore also fulfill a similar role in the cell metabolism, the clustering result can certainly be regarded as more meaningful.

All analyses were performed using the rich internet application QuPE. Results as well as datasets are available online at http://qupe.cebitec.uni-bielefeld.de (see Additional file 8 for a short guide through the data).

\section{Methods}

\section{Proteomics datasets}

For the evaluation and comparison of the different statistical analysis methods we have chosen three different datasets. The first experiment (A) was conducted by Hahne et al. [1]. In a study on Bacillus subtilis wildtype strain $168(\operatorname{trp} C 2)$ the adaption of the organism to salt stress was analyzed at the level of the proteome as well as the transcriptome. Each three samples were grown in ${ }^{15} \mathrm{~N}$-labeled medium and mixed with equal amounts of unlabeled, so to say ${ }^{14} \mathrm{~N}$-labeled, proteins for relative quantification. LC-MS/MS measurements on an LTQ Orbitrap XL (Thermo Fisher Scientific, Bremen) coupled to a nanoAcquity UPLC (Waters) resulted in each 60 raw data files, which were then transformed into the open source format mzXML using the tool "ReAdW" $[54,55]$. It has to be noted that in our experiment only the membrane fraction was investigated, which however also comprises high numbers of cytosolic proteins $(>70 \%,[1])$.

Likewise targeting Bacillus subtilis, Otto et al. performed a comprehensive monitoring of temporal changes in the proteome, the transcriptome and the metabolome as a result of glucose starvation. In this second experiment (B), sample preparation and labeling have been carried out analogous to A, and the experiment also consists of three replicates. Here, only the cytosolic fraction was included in our analysis, which, nonetheless, has the impressive amount of overall 292 raw data files [2].

The third experiment $(C)$ scrutinizes the physiological adaption of Corynebacterium glutamicum to benzoate and glucose each as sole carbon source. Haußmann et al. [3] originally performed SIMPLE [56] digest and MudPIT in combination with metabolic labeling using

${ }^{15}$ Non three replicates and comprehensively investigated the membrane proteome. In this work, however, only one replicate of the predigest fraction was taken into account to demonstrate the applicability of the provided evaluation strategy on smaller datasets. Overall, 22 LCMS/MS runs were considered, all measured using a Accela gradient HPLC pump system coupled to an LTQ Orbitrap (Thermo Fisher Scientific, Bremen).

\section{Identification}

In contrast to the published work, for experiment $\mathrm{A}$ and $B$ data was imported into the rich internet application QuPE [4]. A Mascot (TM) [29] search was conducted using a database that contained the complete proteome of Bacillus subtilis as well as an equally-sized set of randomized amino acid sequences allowing for the later calculation of false discovery rates as suggested by Reidegeld et al. [57]. Peptide tolerance was set to 10.0 $\mathrm{ppm}, \mathrm{ms} / \mathrm{ms}$ tolerance to $1000.0 \mathrm{mmu}$, and two missed cleavage sites were allowed. Oxidation of methionine was allowed as a variable modification, and furthermore, a modification of arginine and lysine was introduced to account for a possible selected non-monoisotopic peak of a ${ }^{15} \mathrm{~N}$-labeled precursor with a weight of approximately $1 \mathrm{Da}$ [58]. Only hits having a score above Mascot's own significance threshold $(p<0.05)$ were kept. In addition, false discovery rates were calculated in QuPE and required to be below $p<0.05$. For each protein at least two peptide hits had to be available, and for each spectrum only one, the best-scoring, hit was selected. In experiment A this resulted in 173,044 peptide hits accounting for overall 1445 proteins. The high number of 620,305 identified peptides was found for experiment B. These constitute 2472 different proteins.

For experiment $\mathrm{C}$, protein identification was based on the original Sequest (TM) [30] search results. The database contained 3058 sequences of Corynebacterium glutamicum. Filter criteria (for further details please refer 
to the original publication) were selected in such a way that a false discovery rate of less than $1 \%$ was achieved. In summary, 12,870 peptide identifications were imported in QuPE which in turn represent 712 proteins.

\section{Quantification}

For all three experiments, quantification was performed using QuPE's built-in algorithm using an ${ }^{15} \mathrm{~N}$ incorporation level of $98 \%$ and under consideration of a peptide's elution in a range of 30 to 60 seconds before and after the scan it was identified in. Rather strict parameters were employed $(r>0.4$, isotopic distribution similarity $>0.8$ ) and results were filtered for a signal-to-noise value of at least 3.0. In summary, for experiment A 58,895 peptides could be quantified accounting for 1285 proteins; in experiment B it were 180,913 peptides amounting to 2321 proteins, and in experiment C 3,699 peptides and 589 proteins. In this regard, one special case has to be highlighted as protein identification in the samples of experiment $\mathrm{A}$ and $\mathrm{B}$ also took into account contaminations by using not only a Bacillus subtilis sequence database but also a set of common laboratory contaminants. Obviously, these proteins were not subject to the labeling, but some showed high signal-to-noise values for the unlabeled peptide. We kept these-actually senseless-proteins in our analysis as they provide a good example for measurements having a high variance. Due to a label swap (control ${ }^{15} \mathrm{~N}$, experiment ${ }^{14} \mathrm{~N}$ ) in one of the samples not only very high but also very low ratios were obtained.

\section{Additional material}

Additional file 1: Statistical analysis. Table showing the ANOVA and Kruskal-Wallis test results of all proteins. Proteins are sorted by the ANOVA's $p$-value in ascending order. The assumption that all values are derived from a normal distribution was investigated using a ShapiroWilks test. To analyze the homogeneity of variances of each group a Fligner-Killeen test was performed. All computed $p$-values where corrected using the method described by Holm (experiment A).

Additional file 2: Statistical analysis. Table showing the ANOVA and Kruskal-Wallis test results of all proteins. Proteins are sorted by the ANOVA's $p$-value in ascending order. The assumption that all values are derived from a normal distribution was investigated using a ShapiroWilks test. To analyze the homogeneity of variances of each group a Fligner-Killeen test was performed. All computed $p$-values where corrected using the method described by Holm (experiment B).

Additional file 3: Statistical analysis. Table showing the ANOVA and Kruskal-Wallis test results of all proteins. Proteins are sorted by the ANOVA's $p$-value in ascending order. The assumption that all values are derived from a normal distribution was investigated using a ShapiroWilks test. To analyze the homogeneity of variances of each group a Fligner-Killeen test was performed. All computed $p$-values where corrected using the method described by Holm (experiment C).

Additional file 4: Cluster analysis and cluster validation. Formal definition of cluster analysis, correlation-based distances and utilized cluster indexes for cluster validation.

Additional file 5: Cluster validation. Comparison of clustering results having equally sized partitions produced by different cluster algorithms using the adjusted Rand index (experiment A).
Additional file 6: Cluster validation. Comparison of clustering results having equally sized partitions produced by different cluster algorithms using the adjusted Rand index (experiment B).

Additional file 7: Cluster validation. Comparison of clustering results having equally sized partitions produced by different cluster algorithms using the adjusted Rand index (experiment C).

Additional file 8: A walk through QuPE. A walk-through guide introducing some of the functionality of the rich internet application QUPE.

\section{Acknowledgements}

The authors wish to thank the BRF system administrators for expert technical support. We acknowledge the funding by the BMBF in the frame of the QuantPro initiative (grant 0313812) and the support for the publication fee by the Deutsche Forschungsgemeinschaft and the Open Access Publication Funds of Bielefeld University.

\section{Author details}

${ }^{1}$ Computational Genomics, Center for Biotechnology (CeBiTec), Bielefeld University, Germany. ${ }^{2}$ Biodata Mining Group, Faculty of Technology, Bielefeld University, Germany. ${ }^{3}$ Bioinformatics Resource Facility, CeBiTec, Bielefeld University, Germany. ${ }^{4}$ Chair for Proteomics and Bioanalytics, Center of Life and Food Sciences Weihenstephan, Technische Universität München, Germany. ${ }^{5}$ Institute of Microbiology, Ernst-Moritz-Arndt-University Greifswald, Germany. ${ }^{6}$ Plant Biochemistry, Ruhr University Bochum, Germany. ${ }^{7}$ Current Address: Chair for Proteomics and Bioanalytics, Center of Life and Food Sciences Weihenstephan, Technische Universität München, Germany.

\section{Authors' contributions}

SPA performed the evaluation and implemented all methods within the rich internet application QPE. HH, AO, UH, AP and DB provided datasets and material, and contributed to the biological background. TWN and AG initiated, supervised, and directed the project. All authors have read and approved the manuscript.

\section{Competing interests}

The authors declare that they have no competing interests.

Received: 9 February 2011 Accepted: 11 June 2011

Published: 11 June 2011

\section{References}

1. Hahne H, Mäder U, Otto A, Bonn F, Steil L, Bremer E, Hecker M, Becher D: A comprehensive proteomics and transcriptomics analysis of Bacillus subtilis salt stress adaptation. J Bacteriol 2010, 192(3):870-882.

2. Otto A, Bernhardt J, Meyer H, Schaffer M, Herbst FA, Siebourg J, Mäder U, Lalk M, Hecker M, Becher D: Systems-wide temporal proteomic profiling in glucose-starved Bacillus subtilis. Nat Commun 2010, 1:137.

3. Haussmann U, Qi SW, Wolters D, Rögner M, Liu SJ, Poetsch A: Physiological adaptation of Corynebacterium glutamicum to benzoate as alternative carbon source - a membrane proteome-centric view. Proteomics 2009, 9(14):3635-3651.

4. Albaum SP, Neuweger $H$, Fränzel B, Lange S, Mertens D, Trötschel C, Wolters D, Kalinowski J, Nattkemper TW, Goesmann A: Qupe-a Rich Internet Application to take a step forward in the analysis of mass spectrometry-based quantitative proteomics experiments. Bioinformatics 2009, 25(23):3128-3134.

5. de Godoy LMF, Olsen JV, Cox J, Nielsen ML, Hubner NC, Fröhlich F, Walther TC, Mann M: Comprehensive mass-spectrometry-based proteome quantification of haploid versus diploid yeast. Nature 2008, 455(7217):1251-1254.

6. Mallick P, Kuster B: Proteomics: a pragmatic perspective. Nat Biotechnol 2010, 28(7):695-709.

7. Gouw JW, Krijgsveld J, Heck AJR: Quantitative proteomics by metabolic labeling of model organisms. Mol Cell Proteomics 2010, 9:11-24.

8. Hufnagel $P$, Rabus R: Mass spectrometric identification of proteins in complex post-genomic projects. Soluble proteins of the metabolically 
versatile, denitrifying 'Aromatoleum' sp strain EbN1. J Mol Microbiol Biotechnol 2006, 11(1-2):53-81.

9. Bantscheff M, Schirle M, Sweetman G, Rick J, Kuster B: Quantitative mass spectrometry in proteomics: a critical review. Anal Bioanal Chem 2007, 389(4):1017-1031.

10. Mueller LN, Brusniak MY, Mani DR, Aebersold R: An assessment of software solutions for the analysis of mass spectrometry based quantitative proteomics data. J Proteome Res 2008, 7:51-61.

11. Zhu H, Pan $\mathrm{S}, \mathrm{Gu} \mathrm{S}$, Bradbury EM, Chen X: Amino acid residue specific stable isotope labeling for quantitative proteomics. Rapid Commun Mass Spectrom 2002, 16(22):2115-2123.

12. Ong SE, Blagoev B, Kratchmarova I, Kristensen DB, Steen H, Pandey A, Mann M: Stable isotope labeling by amino acids in cell culture, SILAC, as a simple and accurate approach to expression proteomics. Mol Cell Proteomics 2002, 1(5):376-386.

13. MacCoss MJ, Wu CC, Liu H, Sadygov R, Yates JR: A Correlation Algorithm for the Automated Quantitative Analysis of Shotgun Proteomics Data. Anal Chem 2003, 75(24):6912-6921.

14. Wolters $D$, Washburn $M$, Yates J: An automated multidimensional protein identification technology for shotgun proteomic. Anal Chem 2001, 73(23):5683-5690.

15. Ellison SLR, Barwick VJ, Farrant TJD: A Practical Statistics for the Analytical Scientist: A Bench Guide 2nd Edition. 2 edition. The Royal Society of Chemistry; 2009

16. Crawley MJ: Statistics - An Introduction using R Wiley 2007.

17. Bacher J: Clusteranalyse. 2 edition. Oldenbourg; 1996.

18. Hastie T, Tibshirani R, Friedman J: The Elements of Statistical Learning Springer Series in Statistics, Springer; 2001.

19. Cormack R: A Review of Classification. Journal of the Royal Statistical Society (Series A) 1971, 134(3):321-367.

20. Everitt BS, Landau S, Leese M: Cluster Analysis. fourth edition. Arnold; 2001

21. Sneath PHA, Sokal RR: Numerical taxonomy - the principles and practice of numerical classification Freeman; 1973.

22. Sokal RR, Michener CD: A Statistical Method for Evaluating Systematic Relationships. The University of Kansas science bulletin 1958, 38:1409-1438.

23. MCQuitty LL: Similarity Analysis by Reciprocal Pairs for Discrete and Continuous Data. Educational and Psychological Measurement 1966, 26:825-831.

24. Forgy E: Cluster Analysis of Multivariate Data: Efficiency versus Interpretability of Classifications. Biometrics 1965, 21:768-769.

25. MacQueen J: Some Methods for Classification and Analysis of Multivariate Observations. In Proceedings of the Fifth Berkeley Symposium on Mathematical Statistics and Probability. Volume 1. Edited by: Cam LML, Neyman J. University of California Pr; 1965:281-297.

26. Bezdek JC: Pattern Recognition with Fuzzy Objective Function Algorithms Kluwer Academic Publishers; 1981.

27. Handl J, Knowles J, Kell D: Computational cluster validation in postgenomic data analysis. Bioinformatics 2005, 21:3201-3212.

28. Halkidi M, Batistakis Y, Vazirgiannis M: Cluster Validity Methods: Part I \& II. SIGMOD Rec 2002, 31(2):40-45.

29. Perkins D, Pappin D, Creasy D, Cottrell J: Probability-based protein identification by searching sequence databases using mass spectrometry data. Electrophoresis 1999, 20(18):3551-3567.

30. Eng JK, McCormack AL, III JRY: An approach to correlate tandem mass spectral data of peptides with amino acid sequences in a protein database. Journal of the American Society for Mass Spectrometry 1994, 5(11):976-989

31. Rocke DM: Design and analysis of experiments with high throughput biological assay data. Semin Cell Dev Biol 2004, 15(6):703-713.

32. Hochberg $Y:$ A sharper Bonferroni procedure for multiple tests of significance. Biometrika 1988, 75(4):800-802.

33. Holm S: A simple sequential rejective multiple test procedure. Scandinavian journal of statistics 1979, 6(2):65-70.

34. Benjamini $Y$, Hochberg $Y$ : Controlling the False Discovery Rate: a Practical and Powerful Approach to Multiple Testing. Journal of the Royal Statistical Society (Series B) 1995, 57:289-300.

35. Shapiro SS, Wilk MB: An Analysis of Variance Test for Normality (Complete Samples). Biometrika 1965, 52(3/4):591-611.

36. Fligner MA, Killeen TJ: Distribution-Free Two-Sample Tests for Scale. J Am Stat Assoc 1976, 71(353):210-213.
37. Spearman C: The Proof and Measurement of Association between Two Things. The American Journal of Psychology 1904, 15:72-101.

38. Cohen J: Statistical power analysis for the behavioral sciences Erlbaum; 1988

39. Tukey JW: Exploratory data analysis Addison-Wesley; 1977.

40. Ward J, Joe H: Hierarchical Grouping to Optimize an Objective Function. J Am Stat Assoc 1963, 58(301):236-244.

41. Martinetz TM, Berkovich S, Schulten KJ: Neural-gas network for vector quantization and its application to time-series prediction. IEEE T Neural Networ 1993, 4(4):558-569.

42. Hubert L, Arabie P: Comparing Partitions. Journal of Classification 1985, 2:193-218.

43. Calinski RB, Harabasz J: A dendrite method for cluster analysis. Communications in Statistics 1974, 3:1-27.

44. Davies DL, Bouldin DW: A cluster separation measure. IEEE T Pattern Anal 1979, 1:224-227.

45. Krzanowski WJ, Lai YT: A Criterion for Determining the Number of Groups in a Data Set Using Sum-of-Squares Clustering. Biometrics 1988, 44:23-34.

46. Yeung K, Haynor D, Ruzzo W: Validating clustering for gene expression data. Bioinformatics 2001, 17:309-318.

47. Maulik U, Bandyopadhyay S: Performance Evaluation of Some Clustering Algorithms and Validity Indices. IEEE T Pattern Anal 2002, 24(12):1650-1654.

48. Giancarlo R, Scaturro D, Utro F: Computational cluster validation for microarray data analysis: experimental assessment of Clest, Consensus Clustering, Figure of Merit, Gap Statistics and Model Explorer. BMC Bioinformatics 2008, 9:462.

49. Tatusov RL, Fedorova ND, Jackson JD, Jacobs AR, Kiryutin B, Koonin EV, Krylov DM, Mazumder R, Mekhedov SL, Nikolskaya AN, Rao BS, Smirnov S, Sverdlov AV, Vasudevan S, Wolf YI, Yin JJ, Natale DA: The COG database: an updated version includes eukaryotes. BMC Bioinformatics 2003, 4(41):1-14.

50. Kanehisa M, Goto S: KEGG: Kyoto Encyclopedia of Genes and Genomes. Nucleic Acids Res 2000, 28:27-30.

51. Eisen MB, Spellman PT, Brown PO, Botstein D: Cluster analysis and display of genome-wide expression patterns. Proc Natl Acad Sci USA 1998, 95:14863-14868

52. Karpievitch Y, Stanley J, Taverner T, Huang J, Adkins JN, Ansong C, Heffron F, Metz TO, Qian WJ, Yoon H, Smith RD, Dabney AR: A statistical framework for protein quantitation in bottom-up MS-based proteomics. Bioinformatics 2009, 25(16):2028-2034.

53. Glass GV, Peckham PD, Sanders JR: Consequences of Failure to Meet Assumptions Underlying the Fixed Effects Analyses of Variance and Covariance. Review of Educational Research 1972, 42(3):237-288.

54. Keller A, Nesvizhskii Al, Kolker E, Aebersold R: Empirical statistical model to estimate the accuracy of peptide identifications made by MS/MS and database search. Anal Chem 2002, 74(20):5383-5392.

55. Nesvizhskii Al, Keller A, Kolker E, Aebersold R: A statistical model for identifying proteins by tandem mass spectrometry. Anal Chem 2003, 75(17):4646-4658.

56. Fischer F, Wolters D, Rögner M, Poetsch A: Toward the complete membrane proteome: high coverage of integral membrane proteins through transmembrane peptide detection. Mol Cell Proteomics 2006, 5(3):444-453.

57. Reidegeld KA, Eisenacher M, Kohl M, Chamrad D, Körting G, Blüggel M, Meyer HE, Stephan C: An easy-to-use Decoy Database Builder software tool, implementing different decoy strategies for false discovery rate calculation in automated MS/MS protein identifications. Proteomics 2008, 8(6):1129-1137.

58. Zhang Y, Webhofer C, Reckow S, Filiou MD, Maccarrone G, Turck CW: A MS data search method for improved $15 \mathrm{~N}$-labeled protein identification. Proteomics 2009, 9(17):4265-4270.

doi:10.1186/1477-5956-9-30

Cite this article as: Albaum et al:: A guide through the computational analysis of isotope-labeled mass spectrometry-based quantitative proteomics data: an application study. Proteome Science 2011 9:30. 